\title{
Peptide Neuromodulation of Synaptic Dynamics in an Oscillatory Network
}

\author{
Shunbing Zhao, ${ }^{1}$ Amir Farzad Sheibanie, ${ }^{2}$ Myongkeun 0 h, ${ }^{3}$ Pascale Rabbah, ${ }^{1}$ and Farzan Nadim ${ }^{1,3}$ \\ ${ }^{1}$ Department of Biological Sciences, Rutgers University, Newark, New Jersey 07102, ${ }^{2}$ Department of Neuroscience, University of Medicine and Dentistry of \\ New Jersey, Newark, New Jersey 07102, and 3 Department of Mathematical Sciences, New Jersey Institute of Technology, Newark, New Jersey 07102
}

\begin{abstract}
Although neuromodulation of synapses is extensively documented, its consequences in the context of network oscillations are not well known. We examine the modulation of synaptic strength and short-term dynamics in the crab pyloric network by the neuropeptide proctolin. Pyloric oscillations are driven by a pacemaker group which receives feedback through the inhibitory synapse from the lateral pyloric (LP) to pyloric dilator (PD) neurons. We show that proctolin modulates the spike-mediated and graded components of the LP to PD synapse. Proctolin enhances the graded component and unmasks a surprising heterogeneity in its dynamics where there is depression or facilitation depending on the amplitude of the voltage waveform of the presynaptic LP neuron. The spike-mediated component is influenced by the baseline membrane potential and is also enhanced by proctolin at all baseline potentials. In addition to direct modulation of this synapse, proctolin also changes the shape and amplitude of the presynaptic voltage waveform which additionally enhances synaptic output during ongoing activity. During ongoing oscillations, proctolin reduces the variability of cycle period but only when the LP to PD synapse is functionally intact. Using the dynamic clamp technique we find that the reduction in variability is a direct consequence of modulation of the LP to PD synapse. These results demonstrate that neuromodulation of synapses involves complex and interacting influences that target different synaptic components and dynamics as well as the presynaptic voltage waveform. At the network level, modulation of feedback inhibition can result in reduction of variability and enhancement of stable oscillatory output.
\end{abstract}

\section{Introduction}

Short-term synaptic dynamics such as facilitation and depression have been shown to play an important role in shaping the output of neuronal networks (Abbott et al., 1997; Tsodyks et al., 2000; Manor and Nadim, 2001; Zucker and Regehr, 2002; Deeg, 2009). Synaptic plasticity also ensues from modification of synaptic strength by neuromodulators (Ayali et al., 1998; Sakurai and Katz, 2003). Neuromodulation of the short-term dynamics, reported in many systems (Bristol et al., 2001; Baimoukhametova et al., 2004; Cartling, 2004; Sakurai and Katz, 2009), is considered a form of metaplasticity and can have complex network consequences (Fischer et al., 1997b); yet, little is known about neuromodulation of synaptic dynamics in the context of network oscillations. Synapses often involve distinct components that act at different time scales or involve spike-mediated, graded or asynchronous release (Warzecha et al., 2003; Otsu et al., 2004; Ivanov and Calabrese, 2006b). We explore how neuromodulation modifies distinct components of a synapse in an oscillatory network

Received July 15, 2011; revised Aug. 8, 2011; accepted Aug. 9, 2011.

Author contributions: S.Z. and F.N. designed research; S.Z., A.F.S., P.R., and F.N. performed research; S.Z., M.O., P.R., and F.N. analyzed data; S.Z. and F.N. wrote the paper.

This work was supported by National Institutes of Health Grant MH-60605. A.F.S. was supported by NINDS Grant 1T32NS051157. We thank Jorge Golowasch, Isabel Soffer, and the anonymous reviewers for their comments on the manuscript.

Correspondence should be addressed to Farzan Nadim, Department of Biological Sciences, Rutgers University, 195 University Avenue, Newark, NJ 07102. E-mail: farzan@njit.edu.

DOI:10.1523/JNEUROSCI.3624-11.2011

Copyright $\odot 2011$ the authors $\quad 0270-6474 / 11 / 3113991-14 \$ 15.00 / 0$ and examine how these modulatory actions shape the combined synapse in the context of network activity.

The crustacean pyloric oscillations are generated in the stomatogastric nervous system (STNS) by a pacemaker group consisting of the gap-junction-coupled anterior burster $(\mathrm{AB})$, pyloric dilator (PD), and (in crabs) lateral posterior gastric (LPG) neurons that burst in synchrony. The sole chemical feedback from the pyloric follower neurons to the pacemakers is the synapse from the lateral pyloric (LP) to the PD neurons, according a key role for this synapse in the regulation of pyloric oscillations (Manor et al., 1997; Mamiya et al., 2003; Weaver and Hooper, 2003). A variety of neuromodulators in the STNS modify the intrinsic properties of individual pyloric neurons (HarrisWarrick et al., 1998; Swensen and Marder, 2000) and the strength and dynamics of synapses among these neurons (Johnson et al., $2005,2011)$. We demonstrate the ability of the modulatory neuropeptide proctolin to alter the strength and unmask novel dynamics in the LP to PD synapse. Proctolin is released by projection neurons in the STNS (Nusbaum et al., 2001) and activates a voltage-gated ionic current in several pyloric neurons (Swensen and Marder, 2001). However, the effect of proctolin on the pyloric synapses has not been previously examined. The LP to PD synapse has both spike-mediated and graded components, as found in other systems (Angstadt and Calabrese, 1991; Pan et al., 2001; Warzecha et al., 2003; Ivanov and Calabrese, 2006b). We characterize the effect of proctolin on the strength and shortterm dynamics of the two components of the LP to PD synapse separately. The graded component of this synapse, which depresses in control conditions (Manor et al., 1997), is enhanced 
and can show facilitation in proctolin, while the spike-mediated component is also strengthened by proctolin. To measure the combined effect of proctolin, we record voltage waveforms of the LP neuron during ongoing activity and use these realistic waveforms in the voltageclamped LP neuron to unmask the contribution of these changes to total synaptic output in biologically realistic conditions. Finally, using the dynamic clamp technique to modify the synaptic current, we show that proctolin reduces variability in pyloric oscillations through its modulation of the LP to PD synapse.

\section{Materials and Methods}

Preparation and identification of the neurons. Experiments were conducted on the STNS of male crabs Cancer borealis. Animals were obtained from local markets and maintained in filtered, recirculating seawater tanks at $10-12^{\circ} \mathrm{C}$. The STNS was dissected out using standard procedures (Blitz et al., 2004; Tohidi and Nadim, 2009). Briefly, the complete isolated STNS [including the stomatogastric ganglion (STG), the oesophageal ganglion (OG), and the paired commissural ganglia $(\mathrm{CoG})$;

Fig. $1 A$ ] was pinned down on a Sylgard-coated Petri dish. The STG was desheathed to facilitate penetration of the pyloric neuron cell bodies. All preparations were continuously superfused with chilled $\left(10-13^{\circ} \mathrm{C}\right)$ physiological Cancer saline containing (in $\mathrm{mm}$ ): $11 \mathrm{KCl}, 440 \mathrm{NaCl}, 13$ $\mathrm{CaCl}_{2}, 26 \mathrm{MgCl}_{2}, 11.2$ Trizma base, 5.1 maleic acid, $\mathrm{pH}=7.4-7.5$. Proctolin (Sigma-Aldrich) was dissolved as stock solution in distilled water to a final concentration of $10^{-3} \mathrm{M}$, divided into aliquots and frozen at $-20^{\circ} \mathrm{C}$. The final concentration was made by dissolving the stock solution in Cancer saline immediately before use. The dose-response effect on the synaptic input-output curve was done by bath-applying proctolin from low to high concentration $\left(10^{-9}-10^{-5} \mathrm{M}\right)$ in $20 \mathrm{~min}$ intervals. All other applications of proctolin were done at $10^{-6} \mathrm{M}$. Proctolin and other solutions were bath applied by means of a switching port in a continuously flowing superfusion system.

Extracellular recordings from identified motor nerves were made using stainless steel wire electrodes, inserted inside and outside of a petroleum jelly well built to electrically isolate a small section of the nerve, and amplified with a Differential AC amplifier (A-M Systems 1700). Intracellular recordings were made from the neuronal cell bodies with sharp glass microelectrodes containing $0.6 \mathrm{M} \mathrm{K}_{2} \mathrm{SO}_{4}$ and $20 \mathrm{~mm} \mathrm{KCl}$ (final electrode resistance 20-30 M $\Omega$ ). Microelectrodes were pulled using a FlamingBrown P-97 micropipette puller (Sutter Instruments). All intracellular recordings were performed in single-electrode current-clamp or twoelectrode voltage-clamp mode (Axoclamp 2B amplifiers; Molecular Devices). Pyloric neurons were identified according to their stereotypical axonal projections in identified nerves and interactions with other STG neurons (Weimann et al., 1991; Blitz et al., 2008).

Effect of proctolin on the strength and dynamics of the LP to PD synapse. Measurements of synaptic output were done by measuring maximum amplitude when there was little variability in the synaptic potential or current amplitudes. In cases where biological or other conditions resulted in variability of synaptic amplitude (such as during ongoing activity) we measured the total area of the IPSP or IPSC.

To study the graded component of the LP to PD synapse, the preparation was superfused with $10^{-7} \mathrm{M}$ tetrodotoxin (TTX; Biotium) to block action potentials and therefore spike-mediated transmission. The LP neuron was two-electrode voltage clamped with a holding potential of $-60 \mathrm{mV}$ and stimulated with multiple square pulses of different amplitudes and fixed $500 \mathrm{~ms}$ duration (and different interpulse intervals as noted in Results), as well as realistic waveforms of different amplitudes and frequencies. Application of realistic waveforms for synaptic measurements was done according to methods we have previously described (Mamiya et al., 2003; Tseng and Nadim, 2010). Graded synaptic responses were used to measure synaptic input-output relationships which were fit with Boltzmann-type equations $1 /\left(1+\exp \left(\left(V_{\mathrm{LP}}-\right.\right.\right.$ $\left.\left.V_{\text {half }} / k_{\text {half }}\right)\right)$.

In almost all recordings, the amplitude of the graded IPSP (gIPSP) did not change subsequent to the fourth pulse and therefore we refer to the gIPSP in response to the fifth pulse as the steady-state gIPSP. To measure short-term synaptic dynamics, the ratio of the fifth to the first mean gIPSP amplitude $\left(A_{5} / A_{1}\right)$ was used. To avoid outliers, the ratio was only calculated if the mean gIPSP amplitude was $\geq 0.5 \mathrm{mV}$. To measure spikemediated transmission, the LP neuron was voltage clamped at a holding potential between -70 and $-50 \mathrm{mV}$ and one of the following two methods was used.

In the first method, short square voltage pulses of fixed $10 \mathrm{~ms}$ duration were used to elicit individual spikes and activate the spike-mediated component of the synapse without eliciting graded release. This was made possible because the voltage-clamp holding potential prevented graded release which depends on changes in the baseline membrane potential (we confirmed that $10 \mathrm{~ms}$ pulses were too short to elicit graded release by showing that no synaptic current was present in the presence of TTX) and, action potentials could be generated by the brief $10 \mathrm{~ms}$ pulses without any significant effect on the baseline membrane potential.

In the second method, antidromic spikes were elicited by stimulating the lateral pyloric nerve (lpn) using a pulse stimulator (A-M Systems isolated pulse stimulator 2100) using $0.5 \mathrm{~ms}, 3-10 \mathrm{~V}$ stimuli. The antidromic spikes (which cannot be clamped with somatic electrodes) invade the arborization of the LP neuron and result in spike-mediated but not graded synaptic release.

Measurement of putative $\mathrm{Ca}^{2+}$ currents was done in the presence of $10^{-7} \mathrm{M}$ TTX and $10 \mathrm{~mm}$ TEA and the presynaptic electrodes were loaded with $2 \mathrm{M}$ TEA and $2 \mathrm{M} \mathrm{CsCl}$ to block potassium currents (B. R. Johnson et al., 2003). To measure presynaptic $\mathrm{Ca}^{2+}$ currents, the experimental protocol was repeated in both normal saline and $\mathrm{Mn}^{2+}$ saline (where $\mathrm{Ca}^{2+}$ in the physiological saline is substituted with $12.9 \mathrm{~mm} \mathrm{Mn}^{2+}$ and $0.1 \mathrm{~mm}$ $\mathrm{Ca}^{2+}$ ) and the difference between the presynaptic currents measured in normal saline and in $\mathrm{Mn}^{2+}$ saline was reported as a putative $\mathrm{Ca}^{2+}$ current (Golowasch et al., 1992). Calcium channel blockers $\mathrm{Ni}^{2+}$ and $\mathrm{Cd}^{2+}$ 
A

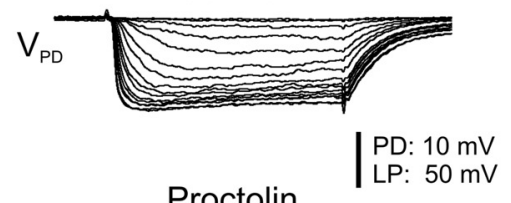

Proctolin

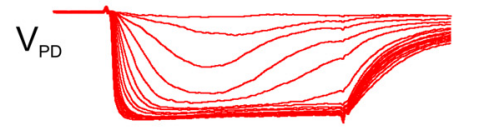

Wash
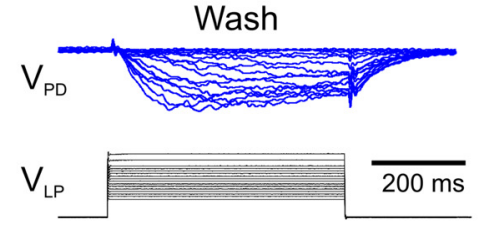

\section{B}

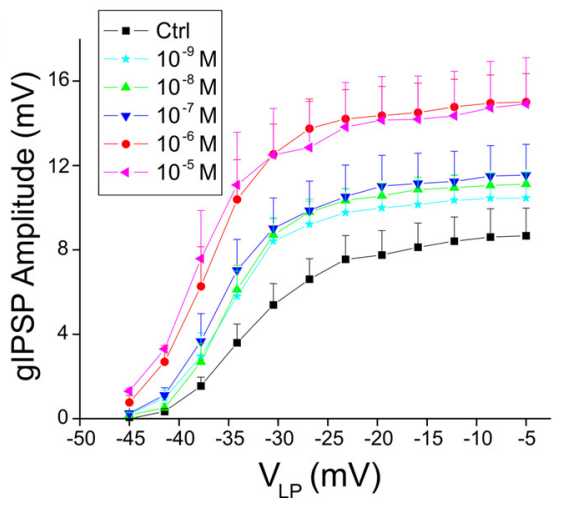

Figure 2. Proctolin strengthens the graded component of the LP to PD synapse. $A$, The $L P$ neuron was voltage clamped at -60 $\mathrm{mV}$ and $500 \mathrm{~ms}$ depolarizing square pulses with increasing amplitudes up to $0 \mathrm{mV}\left(V_{L P}\right)$ were used to activate the $L P$ to $P D$ synapse in Control, $10^{-6}{ }_{M}$ Proctolin and Wash. The resulting graded IPSP (gIPSP) recorded in the PD neuron $\left(V_{P D}\right)$ increased as the amplitude of the $V_{L P}$ pulse increased for all conditions and was always larger in Proctolin. The enhancement of the synaptic strength was reversible after a 45 min wash. The PD neuron was recorded at its resting potential which remains quite stable in TTX. Baseline $V_{P D}(\mathrm{mV})$ : $C \mathrm{Cl},-60.5 ;$ Proc, $-59.5 \mathrm{mV}$; Wash, $-60 \mathrm{mV}$. $\boldsymbol{B}$, The peak amplitudes of gIPSPs were plotted against the $V_{\mathrm{LP}}$ pulse values to obtain the synaptic input- output curves in control and in different doses of proctolin. The amplitudes of gIPSPS were augmented in proctolin in a dose-dependent manner but saturated at $\sim 10^{-6} \mathrm{M}$. $C$, The synaptic input-output curve showed a sigmoidal dependency on the presynaptic voltage for both control and proctolin $\left(10^{-6} \mathrm{M}\right)$. Proctolin significantly strengthened gIPSPs of the LP to PD synapse for presynaptic amplitudes $\geq 35 \mathrm{mV}$ compared with control and wash (two-way ANOVA post hoc Tukey analysis, $p<0.01$ for $V_{\mathrm{LP}}=-35$ to $\left.-10 \mathrm{mV} ; n=6\right)$. $D$, The amplitude of the individual gIPSPs in each preparation was normalized to the maximum gIPSP in that condition. Each data point (black, control; red, proctolin; blue, wash) indicates a single measurement from six preparations, and these measurements were fit with Boltzmann type equations. Proctolin shifted the midpoint of the input- output curve by $\sim 5.5 \mathrm{mV}$ to more negative membrane potentials $\left(V_{\text {half }}\right.$ in mV—Ctrl, $-29.0 \pm$ 0.11; Proc, $-35.4 \pm 0.14$; Wash, $-28.0 \pm 0.44$ ) but did not significantly change the slope of this curve, although the slope became shallower after wash ( $k_{\text {half }}$ in $\mathrm{mV}-\mathrm{Ctrl},-3.11 \pm 0.10$; Proc, $-2.83 \pm 0.12$; Wash, $\left.-6.36 \pm 0.45\right)$.

were bath applied at concentrations of 1 and $0.2 \mathrm{~mm}$, respectively (Golowasch et al., 1992; Hurley and Graubard, 1998; Ivanov and Calabrese, 2006a).

The two PD neurons are anatomically identical and functionally similar; they exhibit similar intrinsic properties and make and receive similar synaptic connections (Miller and Selverston, 1982; Eisen and Marder, 1984; Hooper, 1997; Rabbah et al., 2005; Rabbah and Nadim, 2005; SotoTreviño et al., 2005). For clarity, the figures in this paper only show results from one PD neuron. The two PD neurons were recorded in several preparations for the various protocols to ensure that the responses were similar. In all measurements in this study only the response of a single PD neuron in each preparation is used.

Dynamic clamp. We used the dynamic clamp technique to modify the LP to PD synapse in control saline so as to mimic the effect of the enhancement of the synapse by bath application of proctolin. A NI PCI6070-E board (National Instruments) was used for current injection in dynamic clamp experiments. We use the dynamic clamp software developed in our laboratory (http://stg.rutgers.edu/software) on a Windows platform. In dynamic clamp, ionic currents are calculated using HodgkinHuxley-type equations as described below and continuously updated by recording the membrane potential $(V)$ of the neurons in real time. To modify the synaptic current to mimic the proctolin effect, we injected the difference between the synaptic input-output relationships measured in proctolin and control into the postsynaptic PD neuron. The synaptic strength was calculated by adding the Boltzmann fits to the synaptic input-output curve measured in proctolin (Fig. $2 C, D$, red trace) and subtracting the fit measured in control (Fig. 2C,D, black trace):

$$
\begin{aligned}
I_{\mathrm{dyn}} & =g_{\mathrm{dyn}} s\left(V_{\mathrm{LP}}\right)\left(V_{\mathrm{PD}}-E_{\mathrm{syn}}\right) \\
\frac{d s}{d t} & =\frac{s_{\infty}\left(V_{\mathrm{LP}}\right)-s}{\tau_{\mathrm{s}}} \\
s_{\infty}(V)= & 1.4 /\left(1+\exp \left(-\frac{V+29.0}{2.83}\right)\right) \\
& -1 /\left(1+\exp \left(-\frac{V+35.4}{3.11}\right)\right),
\end{aligned}
$$

where $\tau_{\mathrm{s}}=10 \mathrm{~ms}, g_{\text {dyn }}=100 \mathrm{nS}$, and $E_{\text {syn }}=$ $-80 \mathrm{mV}$.

Recording, analysis, and statistics. Data were acquired using pClamp 9.2 software (Molecular Devices) or the Scope software (available at http://stg.rutgers.edu/software developed in the Nadim laboratory), sampled at $4-5 \mathrm{kHz}$, and saved on a PC using a Digidata 1332A (Molecular Devices) or PCI-6070-E data acquisition board (National Instruments). Statistical and graphical analyses were done using Sigmastat 3.0 (Aspire Software) and Origin 8.0 (OriginLab). Reported statistical significance indicated that the achieved significance level $p$ was below the critical significance level $\alpha=$ 0.05. All error bars shown and error values reported denote SDs.

\section{Results}

During the ongoing pyloric rhythm, the LP and PD neurons fire in alternation (Fig. 1B, left). Removal of descending modulatory inputs to the STG (decentralization) results in slow and irregular pyloric oscillations or the oscillations are completely disrupted (Fig. 1 B, middle; see also Nusbaum and Beenhakker, 2002). As shown in previous studies (Marder et al., 1986; Nusbaum and Marder, 1989a), bath application of proctolin enhances the pyloric rhythm by increasing the amplitude of the slow-wave oscillation of the LP and PD neurons and increasing the spike frequency and number of spikes per burst (Fig. $1 B$, right). It is known that proctolin enhances the bursting activity of the LP and pacemaker neurons by eliciting a voltage-gated inward current (Golowasch and Marder, 1992; Swensen and Marder, 2000). The LP to PD synapse is the only chemical synaptic feedback to the pyloric pacemaker neurons. As such, this synapse is in a key position to affect the frequency and phase relationships of the pyloric network (Eisen and Marder, 1982; Weaver and Hooper, 2003; 


\section{A} $V_{P D}$
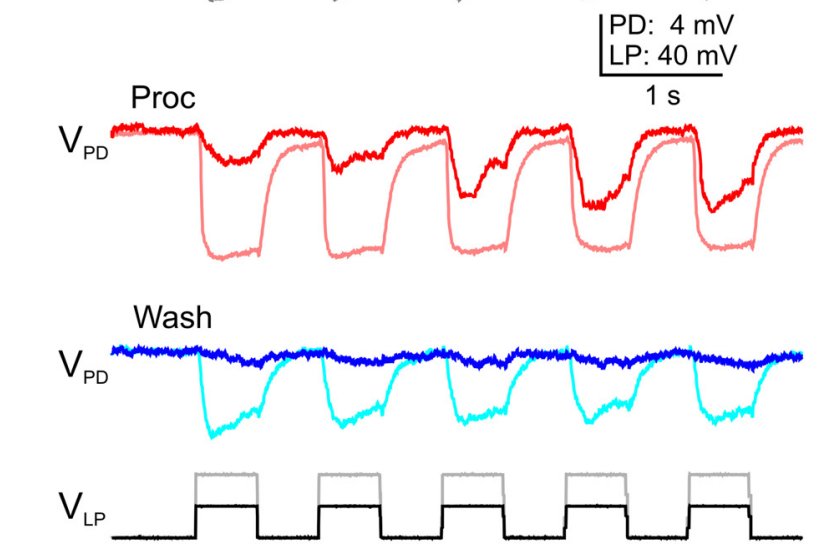

B

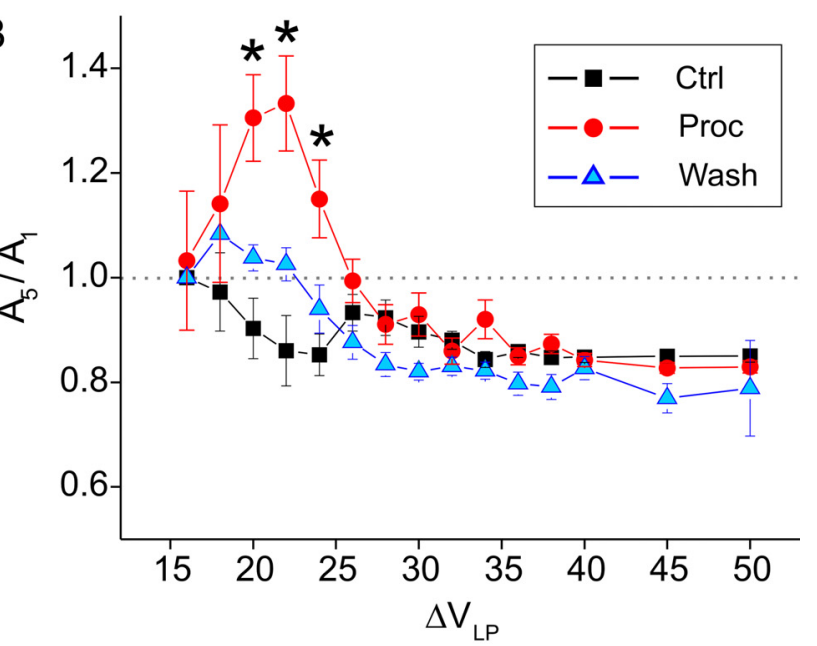

C

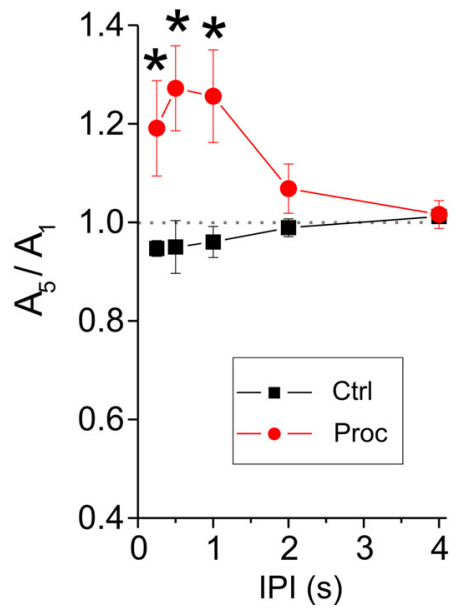

Low-amp $\Delta \mathrm{V}_{\mathrm{LP}}$

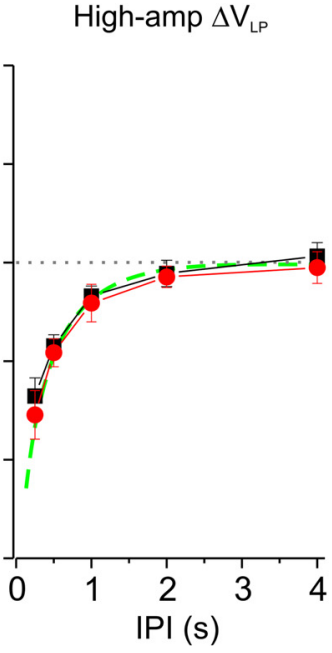

Figure 3. Proctolin modulates the short-term dynamics of the $L P$ to PD graded synapse in response to different amplitude depolarizations. $A, L P$ neuron was voltage clamped at $-60 \mathrm{mV}$ and stimulated with a series of five square pulses of $20 \mathrm{mV}$ ( $V_{\mathrm{LP}}$, black trace) or $40 \mathrm{mV}$ ( $V_{\mathrm{LP}}$ gray trace) amplitudes in control, proctolin, and wash. In control and wash, the LP to PD synapse was depressing. In proctolin, in response to $20 \mathrm{mV}$ presynaptic depolarization, the gIPSPs in the PD neuron showed facilitation. Darker color traces in each condition correspond to the lower pulse amplitude of $20 \mathrm{mV}$. Baseline $V_{P D}$ at $-58 \mathrm{mV}$ in control, $-56.5 \mathrm{mV}$ in proctolin and $-58.5 \mathrm{mV}$ in wash. $B$, The ratio of the fifth to the first mean gIPSP amplitude $\left(A_{5} / A_{1}\right)$ calculated for all presynaptic depolarizations with an interpulse interval of $500 \mathrm{~ms}$ plotted against presynaptic pulse amplitudes $\left(\Delta V_{\mathrm{LP}}\right)$. In control (black), the ratio $A_{5} / A_{1}$ was slightly less than one for all
Mamiya and Nadim, 2004, 2005). Our goal in this study is to characterize the neuromodulation of the LP to PD synapse by proctolin. An examination of the size of the IPSPs resulting from this synapse (gray regions marked in Fig. $1 B$ ) shows that during ongoing oscillations proctolin strengthens the synapse compared with control conditions (Control $1148 \pm 155 \mathrm{mV} \cdot \mathrm{ms}$, Proctolin: $3468 \pm 938 \mathrm{mV} \cdot \mathrm{ms}$; Student's $t$ test, $p<0.01, n=5$ ).

The LP to PD synapse consists of two components: a spikemediated component that manifests as unitary IPSPs in response to individual action potentials in the presynaptic LP neuron (clearly seen in the Decentralized PD neuron trace in Fig. $1 B$ ) and a graded (non-spike-mediated) component (Manor et al., 1997; Ayali et al., 1998; Mamiya and Nadim, 2004). We first examined the effects of proctolin on these two components of LP to PD synapse separately. The input resistance of the LP and PD (measured with $500 \mathrm{~ms}$ voltage-clamp steps from -60 to $-70 \mathrm{mV}$ ) was not significantly altered by bath application of proctolin (PD: ctrl, $10.1 \pm 3.7 \mathrm{M} \Omega$; proc, $11.0 \pm 3.2 \mathrm{M} \Omega$; wash, $9.8 \pm 3.3 \mathrm{M} \Omega$; LP: ctrl, 7.2 $\pm 3.0 \mathrm{M} \Omega$; proc, $8.3 \pm 2.1 \mathrm{M} \Omega$; wash, $8.0 \pm 2.6 \mathrm{M} \Omega$; $n=7$; one-way RM-ANOVA, $p>0.1$ ).

To measure the graded component of the LP to PD synapse, the preparation was bathed with $10^{-7} \mathrm{M}$ TTX to remove action potentials and therefore block spike-mediated transmission. The LP neuron was voltage clamped to a holding potential of $-60 \mathrm{mV}$ and then injected with a series of $500 \mathrm{~ms}$ depolarizing square voltage pulses with increasing amplitudes, in control and during bath application of $10^{-6} \mathrm{M}$ proctolin, and the resulting IPSPs were recorded in the PD neuron (Fig. 2). The IPSP amplitude increased as the amplitude of the LP neuron depolarizations increased in both control and proctolin, as expected from a graded synapse. The amplitudes of the gIPSPs were larger in proctolin, indicating the strengthening of the LP to PD synapse by proctolin. The enhancement of the synaptic strength was reversible after a 45 min wash although the synapse often appeared slower to activate at high presynaptic voltages after wash (Fig. $2 \mathrm{~A}$ ).

The synaptic input-output curve constructed using the peak amplitudes of the gIPSPs showed a sigmoidal dependency on the presynaptic voltage for both control and proctolin (Fig. $2 B, C$ ). The amplitudes of gIPSPs were augmented in proctolin in a dosedependent manner but saturated at $10^{-6} \mathrm{M}$ (Fig. $\left.2 B, n=6\right)$ which is the dose typically used in previous studies of proctolin in this system (Nusbaum and Marder, 1989a; Swensen and Marder, 2000). All subsequent data reported in this study are with $10^{-6} \mathrm{M}$ proctolin.

Proctolin significantly strengthened gIPSPs of the LP to PD synapse for presynaptic amplitudes $\geq 35 \mathrm{mV}$ (two-way ANOVA post hoc Tukey analysis, $p<0.01$ for $V_{\mathrm{LP}}=-35$ to $-10 \mathrm{mV} ; n=$ $6)$. To examine the effect of proctolin on the synaptic inputoutput curve, the amplitude of the individual gIPSPs in each

$\leftarrow$

values of presynaptic pulse amplitudes $\left(\Delta V_{\mathrm{LP}}\right)$, indicating that the synapse was always depressing. In contrast, in the presence of proctolin (red), the $A_{5} / A_{1}$ ratios in response to pulse amplitudes between 20 and $24 \mathrm{mV}$ were $>1$, while the ratios in response to larger pulse amplitudes were $<1$, indicating facilitation and depression, respectively (two-way ANOVA with post hoc Tukey test showing $p<0.001$ between ctl and proctolin and $p<0.008$ between control and wash for $\Delta V_{\mathrm{LP}}=20,22$, and $24 \mathrm{mV}$; not significant at other $\left.\Delta V_{\mathrm{LP}}\right) . C, A_{5} / A_{1}$ measured for different IPI durations in a train of presynaptic pulses applied at low $(20 \mathrm{mV})$ or high $(40 \mathrm{mV})$ amplitudes. Proctolin produced significant facilitation synapse at IPI values $\leq 1000 \mathrm{~ms}$ with low-amplitude presynaptic pulses (two-way ANOVA with post hoc Tukey test showing ${ }^{*} p<0.01$; not significant at other IPI values). With high amplitude pulses there was no statistically significant difference in synaptic dynamics between control and proctolin, both of which resulted in depression (two-way ANOVA; $p>0.5$; dashed green curve shows exponential decay fit $1-0.603 \exp (-t / 434))$. 
preparation was normalized to the gIPSP measured in response to the highest presynaptic amplitude (Fig. 2 D). Each data point in Figure $2 D$ indicates a single measurement in six preparations, and these measurements were fit with Boltzmann type equations (see Materials and Methods). We found that proctolin shifts the midpoint of the input-output curve by $\sim 5.5 \mathrm{mV}$ to more negative membrane potentials ( $V_{\text {half }}$ in $\mathrm{mV}-\mathrm{Ctrl}:-29.0 \pm 0.11$; Proc: $-35.4 \pm 0.14$; Wash: $-28.0 \pm 0.44$; one-way ANOVA, $p<$ $0.001)$ but did not significantly change the slope of this curve, although the slope became shallower after wash $\left(k_{\text {half }}\right.$ in $\mathrm{mV}-$ Ctrl: $-3.11 \pm 0.10$; Proc: $-2.83 \pm 0.12$; Wash: $-6.36 \pm 0.45, p=$ 0.2 for Ctrl vs Wash).

To examine whether proctolin increased the variability in the synaptic amplitudes, we did a two-sample $F$ test for differences in variance between the data measured in control and in proctolin. The test showed that for the non-normalized raw data, there was higher variability in the synaptic amplitude in proctolin than control $\left(F=0.513, p<10^{-9}\right)$. However, when the data were normalized as shown in Figure $2 D$, there was no statistical difference between the two cases $(F=1.029, p=0.79)$ suggesting that the variability was primarily due to changes in amplitude across preparations and not the voltage dependence of the synaptic activation.

The LP to PD synapse has been previously shown to display short-term depression (Manor et al., 1997). To examine whether proctolin modifies the dynamics of this synapse, we injected a train of voltage pulses with different amplitudes from a holding potential of $-60 \mathrm{mV}$ into the LP neuron and recorded the gIPSPs in the PD neuron in control saline and in the presence of $10^{-6} \mathrm{M}$ proctolin. An example of the synaptic response to trains of pulses with two different amplitudes in control and proctolin is shown in Figure 3A. As seen in this figure, in response to $40 \mathrm{mV}$ pulses, the steady-state gIPSP was always smaller than the first pulse gIPSP, indicating synaptic depression, in both control (gray) and proctolin (pink). In contrast, the gIPSPs elicited with $20 \mathrm{mV}$ presynaptic pulses were qualitatively different: in this case, the gIPSPs in the PD neuron either did not change or showed slight depression in control, but facilitation in proctolin: the response to the first pulse was relatively small but became much larger by the third pulse. Figure $3 B$ shows the ratio of the fifth to the first mean gIPSP amplitude ( $A_{5} / A_{1}$; see Materials and Methods) calculated for all presynaptic depolarizations with an interpulse interval (IPI) of $500 \mathrm{~ms}$. In control conditions, the ratio $A_{5} / A_{1}$ was slightly less than one for all values of presynaptic pulse amplitudes $\left(\Delta V_{\mathrm{LP}}\right)$, indicating that the synapse was always depressing. In contrast, in the presence of proctolin, the $A_{5} / A_{1}$ ratios in response to pulse amplitudes between 20 and $24 \mathrm{mV}$ were $>1$ while the ratios in response to larger pulse amplitudes were $<1$, indicating facilitation and depression, respectively (two-way ANOVA with post hoc Tukey test showing $p<0.001$ between control and proctolin and $p<0.008$ between control and wash for $\Delta V_{\mathrm{LP}}=20,22$ and $24 \mathrm{mV}$; not significant at other $\left.\Delta V_{\mathrm{LP}}\right)$. For $\Delta V_{\mathrm{LP}}$ values $<20 \mathrm{mV}$ the gIPSP was typically too small $(<1 \mathrm{mV}$, Fig. $2 C)$ to reliably measure shortterm dynamics which explains the large error bars seen at these voltages. These experiments showed that proctolin acts at the level of the synapse, causing this depressing synapse to become facilitating in response to low-amplitude presynaptic depolarizations, while maintaining a low level of depression with highamplitude depolarizations.

To examine the time dependence of synaptic dynamics, in a separate set of experiments, we changed the IPI duration (250$4000 \mathrm{~ms})$ in trains of presynaptic pulses applied at low $(20 \mathrm{mV})$ or high $(40 \mathrm{mV})$ amplitudes (Fig. $3 C)$. We found that proctolin
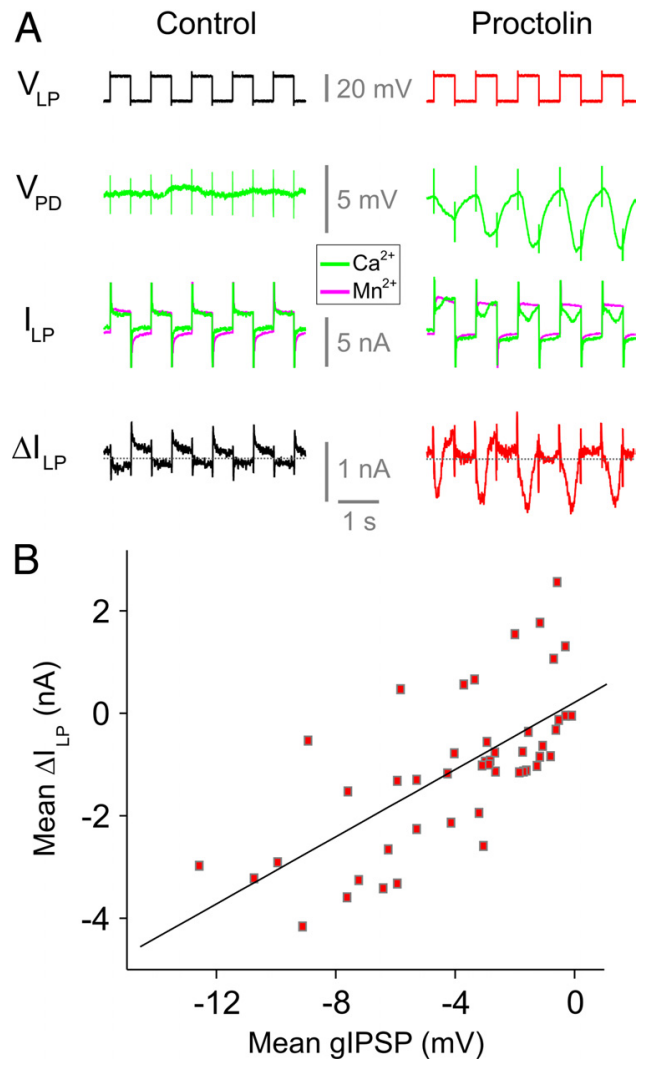

Figure 4. Proctolin caused a facilitation of the LP to PD graded IPSPs in response to lowamplitude presynaptic depolarizing pulses, which is associated with the activation of a $\mathrm{Ca}^{2+}$ like inward current. $\boldsymbol{A}$, The presynaptic currents in the $\mathrm{LP}$ neuron $\left(I_{\mathrm{LP}}\right)$ are measured in $\mathrm{Ca}^{2+}$ saline (green) and in $\mathrm{Mn}^{2+}$ saline (magenta) using a train of low-amplitude presynaptic voltage pulses. The difference between the two measured currents $\left(\Delta I_{\mathrm{LP}}=I_{\mathrm{LP}: \mathrm{Ca}^{2+}}-I_{\mathrm{LP}: \mathrm{Mn}^{2+}}\right)$ reveals a new inward current activated in proctolin (red). A series of low-amplitude presynaptic depolarizing pulses causes little response in postsynaptic neuron PD in control. Proctolin strengthened and caused facilitation in the PD neuron in response to low-amplitude presynaptic depolarizing pulses. $\Delta \mathrm{L}_{\mathrm{LP}}$ also increased with each pulse. $V_{\mathrm{PD}}$ is not shown in $\mathrm{Mn}^{2+}$ because synaptic transmission was blocked (baseline $V_{\mathrm{PD}}$ at $-59 \mathrm{mV}$ control, $-60 \mathrm{mV}$ proctolin; dotted line indicates $0 \mathrm{nA}$ ). $\boldsymbol{B}$, The mean value (area divided by pulse duration) of the presynaptic inward current $\Delta \mathrm{L}_{\mathrm{LP}}$ in response to each pulse plotted against the mean value of the postsynaptic potentials $V_{P D}$ (IPSP area divided by pulse duration) for measurements in $\mathrm{Ca}^{2+}$ saline in proctolin. Linear regression fit shows the correlation between the presynaptic inward current and the postsynaptic potentials $(r=0.69, p<0.001, n=9)$.

produced significant facilitation of the synapse at IPI values $\leq 1000 \mathrm{~ms}$ with low-amplitude presynaptic pulses (two-way ANOVA with post hoc Tukey test showing $p<0.01$ between ctrl and proctolin for IPI $=250,500,1000 \mathrm{~ms}$, not significant at other IPI values; Fig. $3 C$ ). With high-amplitude pulses there was no statistically significant difference in synaptic dynamics between control and proctolin, both of which resulted in depression (twoway ANOVA; $p>0.5)$.

We hypothesized that the appearance of synaptic facilitation with low-amplitude presynaptic voltage pulses in the presence of proctolin was due to additional presynaptic calcium currents. To examine this hypothesis, we applied $20 \mathrm{mV}$ pulses in the LP neuron to correlate the synaptic response with presynaptic $\mathrm{Ca}^{2+}$ currents in the LP neuron. Each experimental protocol was first performed in normal saline and after blocking $\mathrm{Ca}^{2+}$ currents (and therefore synaptic transmission), in both control and proctolin, by substituting the $\mathrm{Ca}^{2+}$ with $\mathrm{Mn}^{2+}$ (see Materials and Methods). In all conditions, we made simultaneous measurements of the presynaptic current (Fig. $4 A, I_{\mathrm{LP}}$ ) and the 
postsynaptic potential (Fig. $4 A, V_{\mathrm{PD}}$ ). The difference between the presynaptic currents measured in normal saline (labeled $\mathrm{Ca}^{2+}$ ) and in $\mathrm{Mn}^{2+}$ saline was measured as a putative calcium current (Fig. $4 A, \Delta I_{\mathrm{LP}}$ ).

In normal saline, there was little synaptic response in control conditions and no apparent synaptic plasticity was observed (Figs. 3A, Ctl black trace; $4 A$, left). In contrast, the synaptic response was strengthened and showed facilitation in proctolin (Figs. $3 A$, Proc, dark red trace; $4 A$, right). For clarity, $V_{\mathrm{PD}}$ is not shown in $\mathrm{Mn}^{2+}$ saline because there was no synaptic transmission in either control or proctolin. The putative calcium current was small in control conditions and its amplitude showed no obvious variation among the different voltage pulses (Fig. $4 \mathrm{~A}$, left, $\left.\Delta I_{\mathrm{LP}}\right)$. In contrast, in the presence of proctolin, this current increased with each subsequent pulse, indicating accumulation of $\mathrm{Ca}^{2+}$ currents (Fig. $4 A$, right, $\left.\Delta I_{\mathrm{LP}}\right)$. We used these data to examine whether the putative $\mathrm{Ca}^{2+}$ current $\Delta I_{\mathrm{LP}}$ measured in proctolin may underlie the increase in the amplitude of the gIPSPs thus resulting in the observed facilitation. The peak of the gIPSPs in response to lowamplitude presynaptic pulses was often highly variable or hard to discern, especially in control where the gIPSPs were small (Fig. $4 A, V_{\mathrm{PD}}$ trace). Due to the variability in the peak current and the peak gIPSPs and the fact that the measured presynaptic currents were at times contaminated with outward currents, we compared the mean values of $\Delta I_{\mathrm{LP}}$ and gIPSP during each pulse. There was a modest positive correlation between the presynaptic inward current and the postsynaptic potentials (Fig. $4 B$, linear fit: $r=$ $0.69, p<0.001, n=9$ ), supporting the hypothesis that the facilitation in proctolin is due to a proctolin-induced accumulation of additional calcium current in the LP neuron.

We also examined whether proctolin affects the strength of the spiked-mediated component of the LP to PD synapse. To elicit single spike-mediated IPSPs (sIPSP), the presynaptic LP neuron was voltage clamped at a holding potential of $-60 \mathrm{mV}$ and stimulated with a very short voltage pulse of duration $10 \mathrm{~ms}$ and amplitude $30 \mathrm{mV}$ to elicit a single action potential (Fig. 5A). The short voltage pulses ensured that there was no graded release (which we also verified by repeating the same protocol in TTX and observing no IPSP; see Materials and Methods). Bath application of proctolin significantly enhanced the amplitude of the unitary sIPSP values (Control: $5.6 \pm 1.1 \mathrm{mV}$, Proctolin: $9.3 \pm 1.0 \mathrm{mV}$; Student's $t$ test, $p<0.001 ; n=6$ ).

It is known that the baseline potential of the presynaptic neuron can have a significant effect on the amplitude of the spikemediated PSPs (Nicholls and Wallace, 1978; Ivanov and
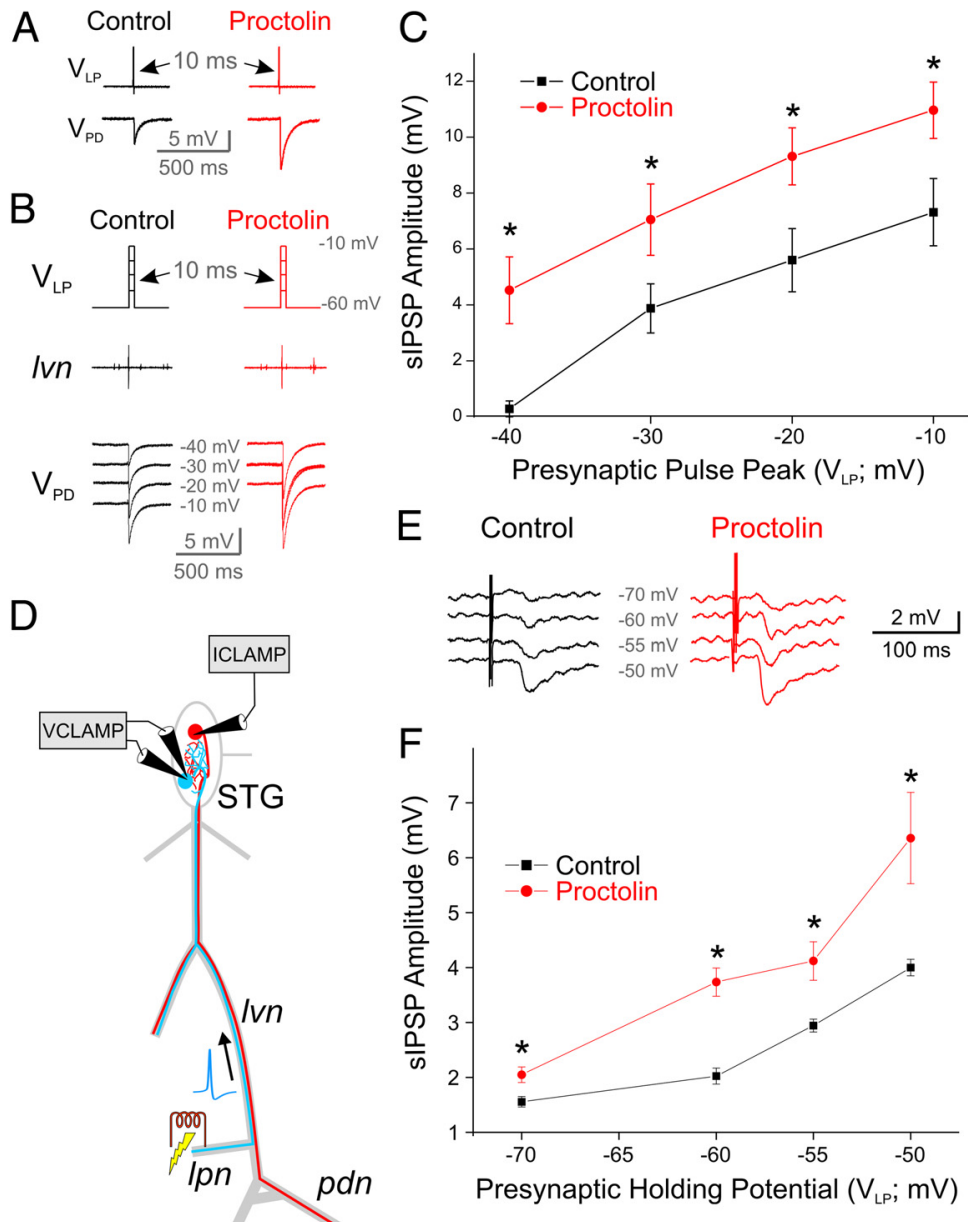

Figure 5. The strength of unitary sIPSPs is enhanced by proctolin. $A$, The LP neuron was voltage clamped at $-60 \mathrm{mV}$ and stimulated with one short square pulse of duration $10 \mathrm{~ms}$ and amplitude $30 \mathrm{mV}$. This resulted in a single action potential in the LP both conditions. $B$, Increasing the presynaptic $10 \mathrm{~ms}$ square pulse amplitude (to peak voltages $V_{\mathrm{LP}}=-40,-30,-20,-10 \mathrm{mV}$, mulus. C, The amplitude of sIPSPs in response to the $10 \mathrm{~ms}$ voltage pulse in control and proctolin increased if the amplitude of this 作 showing how antidromic spikes were elicited in the LPneuron. The LP neuron (blue) soma was voltage clamped and the PD neuron (red) was recorded in current clamp. To elicit antidromic spikes, the Ipn, which contains the axon of the LP neuron but not that of the PD neuron was stimulated. $\boldsymbol{E}$, The $L P$ neuron was voltage clamped at different holding potentials $\left(V_{\mathrm{LP}}=-70,-60,-55\right.$ and $-50 \mathrm{mV}$ ). Thes IPSPs were recorded in PD neuron in responseto antidromic spikes in the $L$ P neuron in control and proctolin $\left(10^{-6} \mathrm{M}\right) . \boldsymbol{F}$, The neuron was increased, the amplitude of SIPSPs in the PD neuron also increased in both control and $10^{-6} \mathrm{M}$ proctolin. In the presence of proctolin, the sIPSPs were larger compared with control (two-way RM-ANOVA, $p<0.05, n=5$ ).

Calabrese, 2003). Although a $10 \mathrm{~ms}$ voltage pulse applied even at $40 \mathrm{mV}$ amplitude in the presynaptic LP neuron never produced any graded synaptic release (IPSP amplitude $0 \mathrm{mV}$ in TTX; $n=$ 3 ), we observed that the amplitude of sIPSPs in response to single action potentials elicited by the $10 \mathrm{~ms}$ voltage pulse in normal saline increased if the amplitude of this short presynaptic voltage pulse was increased (to peak voltages $V_{\mathrm{LP}}=-40,-30,-20,-10$ $\mathrm{mV}$; Fig. $5 B, C$, Control). Additionally, bath application of proctolin significantly increased the sIPSP amplitude for all values of the presynaptic pulse amplitudes (Fig. $5 B, C$; two-way ANOVA post hoc Tukey analysis, $p<0.01, n=8)$.

To completely exclude the possibility that these results were contaminated by the graded component of the LP to PD synapse, we also measured sIPSPs elicited by antidromic spikes while volt- 
A
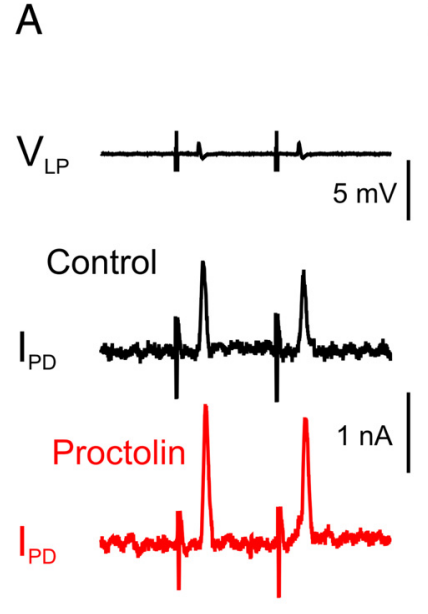

$100 \mathrm{~ms}$
$\mathrm{B}$

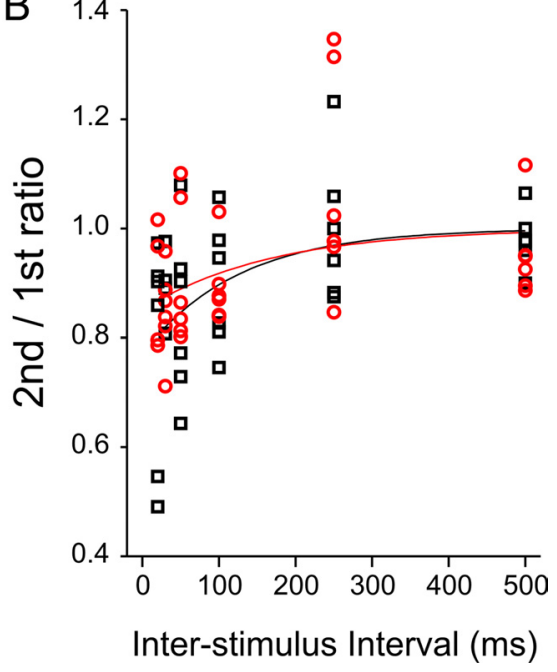

Figure 6. Proctolin does not have a significant effect on the short-term dynamics of the sIPSPs. $A$, The LP and PD neurons were voltage clamped at a holding potential of $-50 \mathrm{mV}$. The resulting spike-mediated synaptic currents in the PD neuron in response to two successive antidromic spikes with a $100 \mathrm{~ms}$ interval were recorded and repeated 10 times. The averaged traces were shown in control (black) and proctolin (red). The sIPSC amplitude was significantly strengthened by proctolin (Ctrl, $0.49 \pm 0.02 \mathrm{nA}$; Proc, $1.3 \pm 0.1 \mathrm{nA}$; Student's $t$ test, $p<0.01, n=4$ ). Baseline $I_{P D}$ at $2.2 \mathrm{nA}$ in control and $2.0 \mathrm{nA}$ in proctolin. $\boldsymbol{B}$, The ratio of the sIPSC amplitudes in response to the first two successive antidromic spikes plotted against interspike interval in control and in proctolin. The exponential fit curves $\left(y=1-A e^{-x / B}\right)$ were plotted in control (black: $A=-0.23, B=125$ ) and in proctolin (red: $A=$ $-0.15, B=167$ ). Proctolin did not cause a significant change in the short-term dynamics compared with control (Student's $t$ test on exponential fits, $p=0.37$ ).

age clamping the LP neuron soma to constant holding potentials in the presence and absence of proctolin. To elicit antidromic spikes, we stimulated the lpn which contains the axon of the LP neuron but not that of the PD neuron (Fig. 5D). The stimulusinduced antidromic spikes propagated back to the STG where they resulted in synaptic release (Fig. 5E). We voltage clamped the LP neuron at different holding potentials below spike threshold $\left(V_{\mathrm{LP}}=-70,-60,-55\right.$, and $\left.-50 \mathrm{mV}\right)$ and recorded the sIPSPs in the PD neuron in control and in the presence of bath-applied proctolin. We found that, as the holding potential of the LP neuron was increased, the amplitude of sIPSPs in the PD neuron also increased in both control and $10^{-6} \mathrm{M}$ proctolin. Additionally, as in the protocol of Figure 5, $B$ and $C$, the sIPSPs were larger in the presence of proctolin compared with control (Fig. 5F; two-way RM-ANOVA, $p<0.05, n=5$ ).

It has been suggested that calcium accumulation through lowthreshold calcium channels activated by subthreshold membrane potential depolarization may result in enough background calcium in the presynaptic neuron to enhance spike-mediated release (Ivanov and Calabrese, 2003). Bath application of $\mathrm{Ni}^{2+}$ is known to block a number of low-threshold calcium channels (Lee et al., 1999; Perez-Reyes, 2003), including those responsible for graded transmission in leech heart interneurons (Ivanov and Calabrese, 2006b). We examined the effects of $\mathrm{Ni}^{2+}$ on the amplitudes of sIPSPs in the LP to PD synapse. We found that sIPSPs (elicited by $10 \mathrm{~ms}$ voltage pulses with different amplitudes similar to Fig. $5 B$ ) were weakened by bath application of $\mathrm{Ni}^{2+}$ by $40-$ $75 \%$ (two-way ANOVA, $p<0.05, n=5$ ) but continued to be enhanced with baseline amplitude (data not shown; Ctrl: from $0.8 \pm 0.6$ at $10 \mathrm{mV}$ amp to $8.5 \pm 1.5 \mathrm{mV}$ at $40 \mathrm{mV}$ amp; $\mathrm{Ni}^{2+}$ : from $0.9 \pm 0.4$ at $10 \mathrm{mV}$ amp to $4.8 \pm 0.4 \mathrm{mV}$ at $40 \mathrm{mV}$ amp; $n=$ 5). It was often difficult to assess the effect of $\mathrm{Ni}^{2+}$ on synaptic transmission because, with long duration bath application, it resulted in membrane potential oscillations reminiscent of an ongoing pyloric rhythm, even in the presence of TTX (Zirpel et al.,

1993). Yet, these results suggest that although an increase in the background calcium in the presynaptic neuron might cause the increase of the sIPSP amplitude as the baseline membrane potential increases, such background calcium is only partially due to $\mathrm{Ni}^{2+}$-sensitive low-threshold calcium channels.

We also examined the effect of proctolin on the short-term dynamics of the sIPSPs. The LP neuron was clamped at a holding potential of $-50 \mathrm{mV}$ and the $\mathrm{lpn}$ was stimulated to produce two successive antidromic spikes, and the interspike intervals (ISIs) were varied (ISI: 30, 50, 100, $250,500 \mathrm{~ms})$. Because the stimulus artifact often interfered with the measurement of the sIPSPs with short ISIs, in this protocol we also voltage clamped the postsynaptic PD neuron with two electrodes at a holding potential of $-50 \mathrm{mV}$ and measured the spike-mediated synaptic currents (sIPSCs) which decay significantly more rapidly than the sIPSPs and are therefore less prone to contamination by the artifacts due to subsequent pulses (especially with short ISIs). The sIPSCs were measured and averaged for 10 repeated trials at each ISI value in each experiment and the resulting average amplitude was used as a single data point. As expected, the sIPSC amplitude was significantly strengthened by proctolin (Ctrl: $0.49 \pm 0.02 \mathrm{nA}$, Proc: $1.3 \pm 0.1 \mathrm{nA}$; Student's $t$ test, $p<$ $0.01, n=4$; Fig. $6 A$ ). To measure the extent of synaptic dynamics, we measured the ratio of the peaks of second to first sIPSC. The sIPSCs of LP to PD synapse exhibited short-term depression in both control and proctolin at shorter ISIs (Fig. 6B). However, there was no difference in depression between control and proctolin (exponential fit curves $y=1-A e^{-x / B}$ : Control $A=0.23$, $B=125$; Proctolin $A=0.15, B=167$; Student's $t$ test, $p=0.37$ ).

So far, our results demonstrated that proctolin can enhance both graded and spike-mediated components of the LP to PD synapse. A natural question is how the total IPSC is affected by proctolin. To explore this question we decentralized the preparation (Fig. 1B), voltage clamped the LP neuron at a holding potential of $-60 \mathrm{mV}$ and applied a voltage profile constructed using a prerecorded realistic LP neuron waveform as we have done in previous studies (Manor et al., 1997; Mamiya and Nadim, 2004; Johnson et al., 2005; Rabbah and Nadim, 2007). The LP waveform was applied to the voltage clamped LP soma periodically with a cycle period of $1 \mathrm{~s}$ and with a total amplitude of $26 \mathrm{mV}$ (as measured in the recording of the waveform) or $39 \mathrm{mV}$ (1.5 times the recorded amplitude). The scaling factor of 1.5 was used because it was the smallest scaling factor of this waveform that consistently resulted in synaptic depression in the gIPSP in both control saline and proctolin. The resulting IPSP was measured in the PD neuron in control saline or in the presence of proctolin (Fig. 7). When the measurements were done in normal saline (i.e., without TTX), the simulated action potentials of the realistic waveform resulted in real action potentials in the presynaptic LP neuron which could be seen on the extracellular recordings of the nerve lvn (Fig. 7A). As a result, the IPSP measured in these conditions was a combination of both graded and spike-mediated components. Additionally, the number and frequency of action 
A Normal Saline -Ctrl B TTX - Ctrl
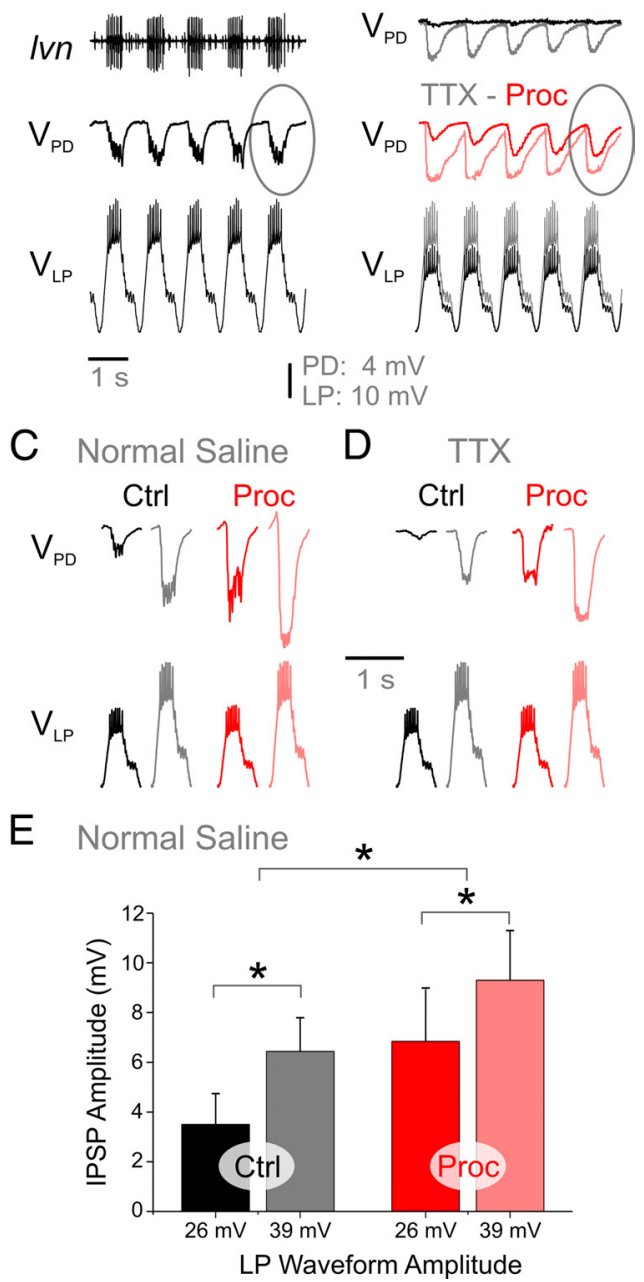

Figure 7. Proctolin strengthens the total LP to PD synaptic IPSP measured using prerecorded realistic waveforms in the $L P$ neuron. $A$, After decentralizing the preparation, the $L P$ neuron was voltage clamped at a holding potential of $-60 \mathrm{mV}$ and injected with a voltage profile constructed using a prerecorded realistic $L P$ neuron waveform $\left(V_{\mathrm{LP}}\right)$ with a total amplitude $39 \mathrm{mV}$ (1.5 times the recorded amplitude). The resulting IPSP was measured in the PD neuron $\left(V_{\mathrm{PD}}\right)$. The simulated action potentials of the realistic waveform resulted in real action potentials in the presynaptic $L P$ neuron which were recorded on the nerve lvn. Baseline $V_{P D}$ at $-59 \mathrm{mV}$ in Normal Saline control and $-60 \mathrm{mV}$ in both TTX control and TTX proctolin. Voltage scale bar applies to $\boldsymbol{A}-\boldsymbol{D}$. $\boldsymbol{B}$, Application of the realistic waveform in TTX with total amplitude of $26 \mathrm{mV}$ (same as recorded amplitude; gray $V_{\mathrm{LP}}$ trace) resulted in synaptic facilitation of the gIPSP in proctolin (red $V_{P D}$ trace) but not control saline (black $V_{P D}$ trace). In contrast, application of the waveform at $39 \mathrm{mV}$ (gray) resulted in slight depression of the gIPSP both in control (gray) and in proctolin (pink). C, In normal saline, the LP waveform was applied periodically with total amplitude of 26 or $39 \mathrm{mV}$. The resulting IPSP, a combination of the graded and spike-mediated components, was measured in the PD neuron in control saline or in the presence of proctolin. The responses shown are after at least four cycles of the presynaptic waveform (circled in $A$ and $\boldsymbol{B}$ ) when the IPSP has reached its stationary state. $\boldsymbol{D}, \operatorname{In}$ TTX, the same $L P$ waveform was applied periodically with total amplitude of 26 or $39 \mathrm{mV}$. The resulting IPSP in this case consists only of the graded component. All responses shown in $\mathbf{C}$ and $\boldsymbol{D}$ were measured in the same preparation. $E$, Mean values of the IPSP as shown in (indicate a significant increase with both amplitude and Proc application (two-way RM-ANOVA; $p<0.05, n=5 ;{ }^{*} p<0.05$ in posthoc Tukey analysis). Ctrl indicates control saline. In $\boldsymbol{B}-\boldsymbol{E}$, darker colors in each condition correspond to the lower amplitude waveform (26 mV).

potentials were the same in control and proctolin because each (simulated) action potential of the prerecorded waveform elicited an action potential in the LP neuron.

To examine how much of the total IPSP was due to the graded component in the different conditions we repeated these mea- surements in TTX. In these conditions, action potentials were blocked and therefore the spike-mediated IPSP was removed. Note that the simulated action potentials of the prerecorded waveform were still artificially played back in the voltage clamped LP neuron but, in TTX, these do not result in biological action potentials. Application of the realistic waveform in TTX with total amplitude of $26 \mathrm{mV}$ (same as recorded amplitude) resulted in synaptic facilitation of the gIPSP in proctolin (Fig. $7 B$, red $V_{\mathrm{PD}}$ trace) but not control saline (black $V_{\mathrm{PD}}$ trace). In contrast, application of the waveform at $39 \mathrm{mV}$ resulted in depression of the gIPSP both in control (gray) and in proctolin (pink). The effect of presynaptic waveform amplitude on synaptic dynamics is similar to that reported with the pulse waveforms shown in Figure 4.

We compared the IPSP amplitudes after at least five cycles of the realistic waveform application (circled region in Fig. $7 A, B$ ) to avoid measuring the transients. Increasing the amplitude of the presynaptic waveform resulted in a larger total IPSP in the PD neuron and proctolin always increased the synaptic amplitude in each preparation (Fig. $7 C$ ). On average, both waveform amplitude and the presence of proctolin significantly increased the total synaptic IPSP amplitude (Fig. 7E; two-way RM-ANOVA, $p<0.05, n=5$ ). In TTX, the realistic waveform at $26 \mathrm{mV}$ amplitude did not produce a significant gIPSP in control saline but did so when the amplitude was increased. Additionally, proctolin increased the amplitude of the gIPSP measured with the realistic waveform (data not shown; two-way RM-ANOVA, $p<0.05, n=$ 5). Three conclusions can be derived from these results. First, under all conditions proctolin enhances the synaptic strength; second, in control conditions, but not in proctolin, synaptic release is mostly due to the spike-mediated component and; third, in proctolin compared with control saline, a large increase in the amplitude of the LP neuron waveform has a smaller effect on synaptic strength. This latter conclusion is consistent with the fact that in proctolin the gIPSP depresses with high-amplitude presynaptic input but facilitates with low-amplitude input (Fig. 7B).

Proctolin increases the amplitude of the LP waveform oscillations, but not by a factor of 1.5. To examine the effect on the synaptic output of the change in waveform amplitude and shape induced by proctolin under more realistic biological conditions, we used prerecorded LP neuron waveforms (Fig. $8 \mathrm{~A}$ ) recorded in control and proctolin to voltage clamp the LP neuron. Both waveforms were applied from a minimum voltage of $-60 \mathrm{mV}$ at the amplitudes they were recorded (Fig. 8A) and the resulting combined spike-mediated and graded IPSP was measured in the PD neuron in control saline and after bath application of proctolin. As expected, either waveform produced a significantly larger synaptic output in proctolin than control. However, the different waveforms produced similar synaptic outputs in control conditions but the proctolin waveform produced a significantly larger synaptic output in proctolin (Fig. $8 B$; two-way RM-ANOVA with post hoc Tukey analysis, $p<0.02, n=5$ ). The average increase of the IPSP amplitude by proctolin was $>400 \%$ whereas the increase due to the change of the waveform shape and amplitude in proctolin was only $36 \%$.

Although our characterization of the components of the LP to PD synapse under controlled conditions where we prescribe the presynaptic waveform provides insight into the actions of the modulator proctolin on this synapse, the effect of proctolin on this synapse should also be characterized under more natural biological conditions. As a first step in exploring these more biologically realistic effects, we measured the LP to PD synaptic currents during ongoing pyloric oscillations. To measure the 
A

B

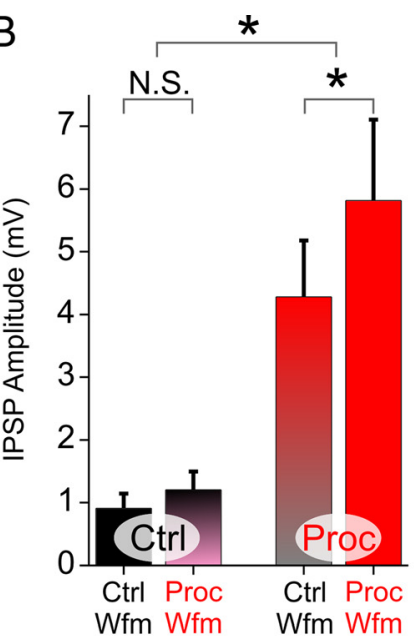

C

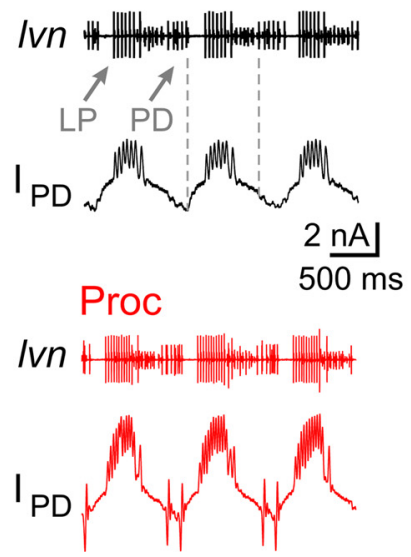

D 5

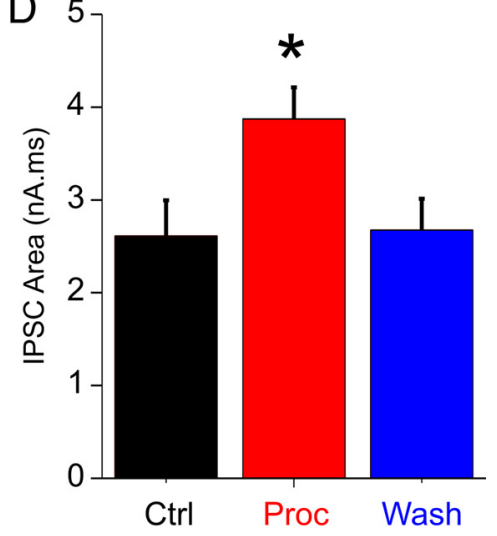

Figure 8. Proctolin effects on the LP to PD synapse in biological conditions. $A$, The LP neuron voltage waveform changes in shape and amplitude in proctolin. These waveforms were played back periodically at the original recorded amplitude (as shown) from a minimum holding potential of $-60 \mathrm{mV}$ and a cycle period of $1 \mathrm{~s}$ into the voltage-clamped $L P$ neuron and the postsynaptic response (mean value) was measured in the PD neuron after reaching stationary state (Fig. 7). B, Bath application of Proc significantly enhanced the IPSP amplitude measured with either waveform (shown in A) compared with control saline (Ctrl). The proctolin-recorded waveform produced a modestly larger response only in the presence of proctolin but not in control saline (two-way RM-ANOVA with post hoc Tukey analysis; ${ }^{*} p<0.02 ; n=5$ ). C, Proctolin enhances the LP to PD synapse during the ongoing pyloric rhythm. The traces of extracellular recordings from Ivn show the ongoing rhythm activity of the pyloric network in control and in proctolin $\left(10^{-6} \mathrm{M}\right)$. One of the two $\mathrm{PD}$ neurons was voltage clamped at a holding potential of $-55 \mathrm{mV}$; the resulting synaptic currents were recorded in this PD neuron in control and in proctolin. Baseline $I_{P D}$ at $-1 \mathrm{nA}$ in control and proctolin. $\boldsymbol{D}$, The synaptic response in PD neuron was integrated from the end of a PD burst to the start of the following PD burst (vertical dashed lines in $\boldsymbol{A}$ ). When necessary, DC current was injected in the second PD neuron to bring the cycle period to $1 \mathrm{~s}$ in all measurements. The IPSC area was significantly larger in proctolin than control or wash (one-way RM-ANOVA, $p<0.01, n=5$ ).

biological IPSCs, one of the two PD neurons was voltage clamped at $-50 \mathrm{mV}$ during ongoing pyloric activity and the IPSCs were measured in control and proctolin (Fig. $8 \mathrm{C}$ ). Note that clamping the PD neuron at a holding potential of $-60 \mathrm{mV}$ stopped the ongoing pyloric rhythm because the PD neurons and the pacemaker $\mathrm{AB}$ neuron are electrically coupled. However, holding the voltage-clamped $\mathrm{PD}$ neuron at $-50 \mathrm{mV}$ did not disrupt the rhythm but often resulted in a slower cycle period. We matched the pyloric cycle period between control and proctolin conditions (compare the extracellular recordings from lvn) by injecting, when necessary, a small DC current in the second PD neuron to speed up or slow down the oscillation to a cycle period of $1 \mathrm{~s}$. This ensured that the comparison in synaptic strength in the two conditions was not due to cycle-period-dependent factors such as short-term depression. The synaptic response in the PD neuron was measured by integrating $I_{\mathrm{PD}}$ from the end of the PD burst to the start of the consequent PD burst (Fig. 8C, vertical dashed line). As expected, the total biological IPSC strength was signifi- cantly larger in proctolin than control or wash (one-way RM-ANOVA, $p<0.01$, $n=5$, Fig. $8 D$ ).

An important functional question is how the enhancement of the LP to PD synapse by proctolin affects the pyloric rhythmic activity. It is difficult to dissect out the contribution of individual factors targeted by proctolin during an ongoing pyloric rhythm because, in addition to modulating the pyloric synapses, proctolin also modulates a voltage-gated current in pyloric neurons (Golowasch and Marder, 1992; Swensen and Marder, 2000). The effect of proctolin bath application on the pyloric cycle period and the activity phases of pyloric neurons has been previously reported (Nusbaum and Marder, 1989b). However, it is unknown to what extent these effects are due to the proctolin modulation of the LP to PD synapse. We were also interested in the effect of proctolin on the variability of the pyloric cycle period. In a previous study, we reported that the LP to PD synapse acts to stabilize the pyloric cycle period: the coefficient of variation (CV) of cycle period is significantly smaller in the presence of the synapse than when the synapse is blocked or functionally removed by hyperpolarizing the LP neuron (Nadim et al., 2011). The results of this previous report were obtained in control saline, but it is reasonable to assume that proctolin enhancement of the LP to PD synapse would further stabilize the pyloric rhythm.

A comparison between the CV of cycle period in control and after bath application of proctolin showed that the CV was indeed significantly decreased in proctolin (Fig. 9A). To find out the extent to which this decrease was due to the enhancement of the synapse, we introduced an extrinsic noisy input to the PD neuron and measured the CV of cycle period in control saline and proctolin during ongoing activity and after the LP to PD synapse was functionally removed by hyperpolarizing the LP neuron $(-5 \mathrm{nA}$ DC current to move the LP membrane potential below $-80 \mathrm{mV}$ ). This noisy input was introduced as 0.5 $\mathrm{nA}, 20 \mathrm{~ms}$ current pulses injected into the PD neuron at discrete time intervals following a Poisson random distribution with a mean frequency of $5 \mathrm{~Hz}$ and was meant to mimic fast projectionneuron input to pyloric pacemaker neurons (Norris et al., 1996) or antidromic spikes from the secondary spike-generation zone of the pacemaker AB neuron (Blitz and Nusbaum, 2008). There was no significant difference in the pyloric cycle period in the presence of the noisy input in these four conditions (one-way RM-ANOVA, $n=6, p>0.1$; data not shown) and, as expected from our previous report, the $\mathrm{CV}$ of cycle period was significantly smaller when the LP to PD synapse was intact, both in control and in proctolin (Fig. 9B). Interestingly, the CV of cycle period was significantly smaller in proctolin compared with control but only when the LP to PD synapse was intact. There was no significant effect 
A
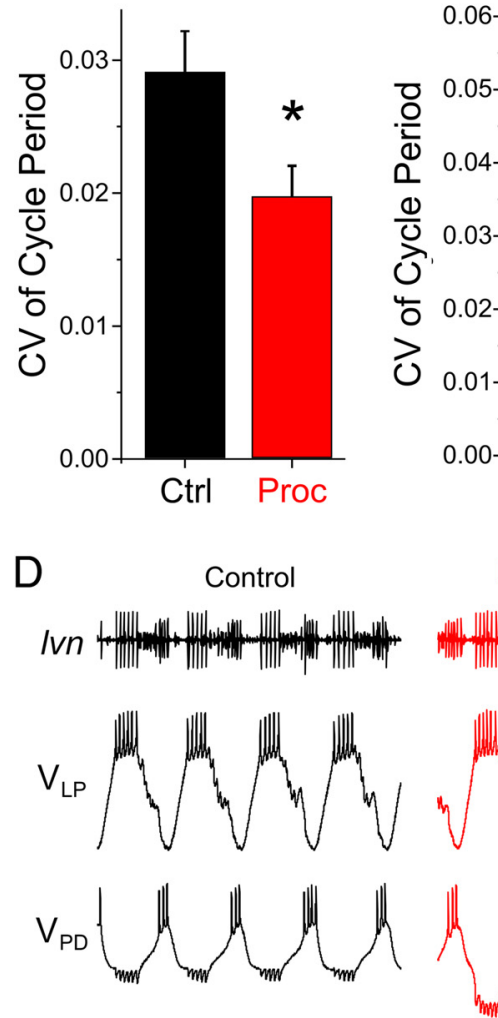

$I_{\text {dyn }}$
B

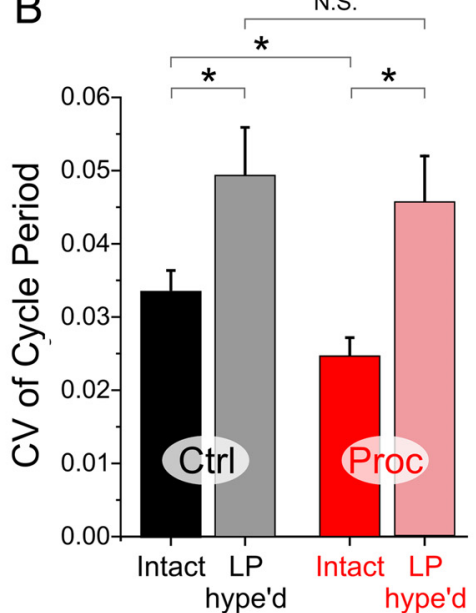

C

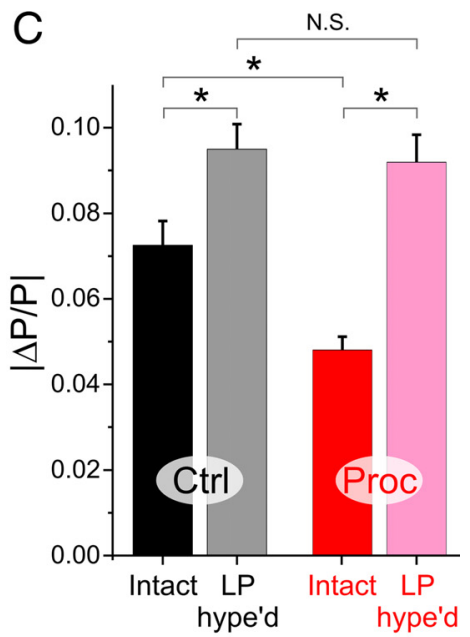

Dynamic clamp
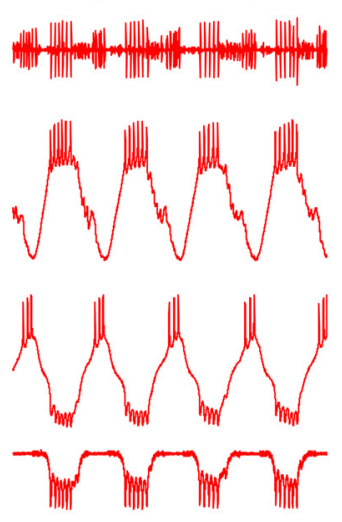

$\mathrm{E}$

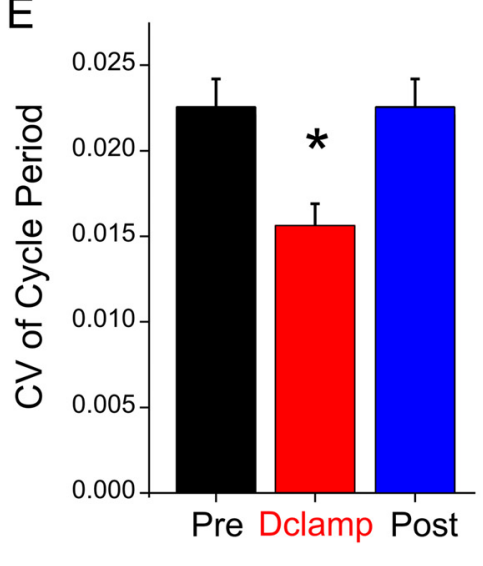

Figure 9. The enhancement of the LP to PD synapse by proctolin reduces the variability in the pyloric cycle period. $\boldsymbol{A}$, The CV of cycle period is significantly reduced in the presence of bath applied proctolin (Student'st test, $p=0.030, n=8)$. $\boldsymbol{B}$, The PD neuron was subjected to a noisy current input ( $0.5 \mathrm{nA}, 20 \mathrm{~ms}$ voltage pulses applied at discrete time intervals following a Poisson distribution with mean frequency of $5 \mathrm{~Hz}$ ). Functional removal of the $L P$ to $P D$ synapse $(-5 \mathrm{nADC}$ current in $\mathrm{LP})$ significantly increased the $\mathrm{CV}$ in both control and proctolin. The $C V$ in proctolin was significantly smaller than control only when the LP to PD synapse was functionally intact (two-way RM-ANOVA with post hoc Tukey analysis; ${ }^{*} p<0.05 ; n=6$ ). C, The pyloric cycle period was perturbed with a single $2 \mathrm{nA}, 50 \mathrm{~ms}$ pulse was injected into the PD neuron once very 10 or so cycles at different phases of the cycle. The absolute value of the change in cycle period $(|\Delta P / P|)$, independent of the stimulus phase, was compared in different conditions. Functional removal of the $L P$ to $P D$ synapse significantly increased $|\Delta P / P|$ in both control and proctolin. The value of $|\Delta P / P|$ in proctolin was significantly smaller than control only when the LP to PD synapse was functionally intact (two-way RM-ANOVA with post hoc Tukey analysis; ${ }^{*} p<0.05 ; n=7$ ). $\boldsymbol{D}$, Adding the proctolin-induced enhancement of the LP to PD synapse using dynamic clamp. Traces of extracellular recordings from Ivn show the ongoing pyloric rhythm activity. Intracellular traces recorded in LP and PD neurons show the rhythmic alternation of the PD and LP neurons. Left panel (black) shows pyloric activity in Control. Using dynamic clamp, the LP to PD synapse was enhanced (right panel, red; see Materials and Methods for equations). $E$, The $C V$ of cycle period was calculated over 40 consecutive cycles from the nerve pdn to measure the effect of the dynamic-clamp enhanced synapse. The variability of the pyloric rhythm period was significantly reduced when the LP to PD synapse was enhanced (Student's $t$ test, $p=0.022, n=5$; experiments in $\boldsymbol{A}-\boldsymbol{C}$ and $\boldsymbol{E}$ are nonoverlapping).

on the CV in proctolin compared with control when the LP neuron was hyperpolarized (Fig. 9B; two-way RM-ANOVA, $n=6$ ).

In a separate set of experiments, we used a different perturbation of the pyloric oscillation by injecting a single brief current pulse ( 2 $\mathrm{nA}, 50 \mathrm{~ms}$ ) at different phases of the cycle and measured the change in cycle period compared with the average of the previous three cycles $(\Delta P / P$, as used to measure the phase-response curve). We found a similar result that this perturbation affected the cycle period significantly more $(|\Delta P / P|$ was larger $)$ when the LP neuron was hyperpolarized (thus the LP to PD synapse was functionally removed) in both control saline and proctolin and the effect of the perturbation was significantly larger in proctolin compared with control saline only when the LP to PD synapse was intact (Fig. 9C). Together, these data imply that the effect of proctolin in stabilizing the pyloric cycle period is due to its enhancement of the LP to PD synapse.

To examine this prediction directly, we used the dynamic clamp technique to modify the LP to PD synapse by incorporating the enhancing effects of proctolin (see Materials and Meth- ods) during ongoing oscillations in control saline. An example of the dynamic-clamp-enhanced LP to PD synapse is shown in Figure $9 D$. We found that the enhanced synapse did not significantly change the activity phase of pyloric neurons or the duty cycle or the PD neuron and resulted in a very small but significant increase in cycle period (from $720 \pm 74 \mathrm{~ms}$ to $746 \pm 72 \mathrm{~ms}$; paired Student's $t$ test, $p<0.05, n=5)$. However, as expected, the enhancement of the synapse with dynamic clamp resulted in a significant reduction in the $\mathrm{CV}$ of the pyloric cycle period (Fig. $9 B$; paired Student's $t$ test, $p<0.05, n=5$ ).

\section{Discussion}

Neuromodulation of synaptic output during ongoing network activity depends on modifications of the activity patterns, synaptic strength and its short-term dynamics. We show that the strength and short-term dynamics of the LP to PD synapse in the oscillatory crab pyloric network are modulated by the neuropeptide proctolin. By implementing the modification of the synaptic 
strength using dynamic clamp we find that the enhancement of this feedback synapse to the pyloric pacemaker neurons reduces the variability in the pyloric cycle period, a result that is also supported by direct measurements of pyloric rhythm variability in the presence of proctolin.

\section{Modulatory actions of proctolin}

Several released neuromodulatory peptides are known to elicit distinct pyloric rhythmic patterns (Marder and Thirumalai, 2002 ) and their network actions have been found to be dose- and frequency-dependent (Nusbaum, 2002). Among the best studied of these neuropeptides is proctolin: all proctolinergic modulatory projection neurons in the crab STNS are known and the network actions of many of these neurons have been characterized (Nusbaum and Beenhakker, 2002). Proctolin strongly excites and modifies the pyloric rhythm (Marder et al., 1986; Hooper and Marder, 1987; Nusbaum and Marder, 1989b), an effect that is at least partly due to the activation of a voltage-dependent nonspecific cation channel in pyloric neurons (Golowasch and Marder, 1992; Swensen and Marder, 2001). However, the effect of proctolin on the pyloric synapses has not been previously examined.

Proctolin exerts its effects through binding to G-protein coupled receptors and subsequently activating downstream signaling pathways (E. C. Johnson et al., 2003). Studies in insect and crayfish muscle have shown that using second messengers $\mathrm{IP}_{3}$ and cAMP, proctolin can increase intracellular calcium concentrations by modulating voltage-dependent or independent channels (Baines et al., 1990; Bishop et al., 1991; Wegener and Nässel, 2000). Proctolin, however, does not appear to cause an increase of cAMP levels in STG neurons (Flamm et al., 1987; Hempel et al., 1996) and little is known about the second messenger pathways underlying the modulatory actions of proctolin in the STNS.

\section{Neuromodulation of short-term synaptic dynamics}

Proctolin unmasks an amplitude-dependent heterogeneity in the short-term dynamics of the LP to PD synapse: the graded component remains depressing with high-amplitude presynaptic stimuli but becomes facilitating with low-amplitude stimuli (Fig. 3 ). While the effects of modulators on synaptic dynamics are documented (Fischer et al., 1997a; Gil et al., 1997; Bristol et al., 2001; Logsdon et al., 2006; Sakurai et al., 2006; Giocomo and Hasselmo, 2007; Parker and Gilbey, 2007; Sakurai and Katz, 2009), only a few studies have shown a modulator to switch the direction of synaptic dynamics from depression to facilitation (Parker, 2003; Baimoukhametova et al., 2004; Bevan and Parker, 2004; Barrière et al., 2008). The switch in synaptic dynamics in these studies has been proposed to be a modulator-dependent change in the initial probability of release $p$ from high (depression) to low (facilitation). The proctolin-induced facilitation of the LP to PD synapse is different from these examples of classical synaptic facilitation which depend on the timing of the presynaptic activity and not its amplitude. Yet a similar mechanism may be at work in the proctolin modulation of the LP to PD synapse as well. Due to the graded nature of this synapse, however, the value of $p$ in the presence of proctolin may depend on the amplitude, not frequency, of the presynaptic stimulus.

Short-term facilitation is generally governed by the properties of the presynaptic neuron (Zucker and Regehr, 2002). Our results also support a partly presynaptic mechanism because the increase in the synaptic strength in response to a train of pulses is correlated with the slow activation of a presynaptic $\mathrm{Mn}^{2+}$-sensitive inward current (Fig. 4). This current is likely to be a calcium current activated by proctolin, although a definitive proof requires additional experiments beyond the scope of this study. Proctolin actions on the LP to PD synapse may be due to the modulation of a low-threshold voltage-gated calcium current. Yet, other mechanisms may underlie the appearance of the putative calcium current. For example, as mentioned above, proctolin activates a nonspecific cation channel in pyloric neurons which is presumed to have a pore block by calcium ions (Golowasch and Marder, 1992; Swensen and Marder, 2000). It has been shown that ions that produce pore block-such as $\mathrm{Mg}^{2+}$ in NMDA channels - can also permeate into the cytoplasm (Stout et al., 1996). This allows the possibility that the proctolin-activated channel has some permeability for calcium, whose accumulation leads to the observed synaptic facilitation. These two hypothetical mechanisms could be distinguished by imaging calcium entry in the presynaptic LP neuron in the presence or absence of calcium channel blockers.

\section{The role of baseline membrane potential on spike-mediated transmission}

The strength of the spike-mediated component of the LP to PD synapse is dependent on the baseline presynaptic membrane potential from which action potentials are generated (Fig. 5). Such a relationship has been shown in many systems including mammalian central synapses (Nicholls and Wallace, 1978; Ivanov and Calabrese, 2003; Alle and Geiger, 2006; Ivanov and Calabrese, 2006b; Shu et al., 2006). In leech heart interneurons, it is known that low-threshold calcium currents activate the graded component of synaptic release, whereas spike-mediated synaptic transmission is triggered by high-threshold calcium currents (Angstadt and Calabrese, 1991; Lu et al., 1997). In these neurons, an increase in the background calcium levels at the presynaptic site due to the membrane potential depolarization is correlated with the increase in the amplitude of the spike-mediated IPSPs (Ivanov and Calabrese, 2006b). We suspect a similar mechanism to be at work for the LP to PD synapse although, unlike the leech synapses where $\mathrm{Cd}^{2+}$ specifically blocks the high-threshold calcium current and thus only spike-mediated release, $\mathrm{Cd}^{2+}$ blocks both components of the LP to PD synapse and cannot be used to test this prediction. The modulatory enhancement of the spikemediated component by proctolin without any effect on the short-term dynamics may be due to an increase in the background calcium levels by proctolin modulation of voltage-gated channels (see above) or calcium release from internal sources.

\section{The effect of proctolin on combined transmission using realistic waveforms}

Graded transmission is the major form of synaptic communication among pyloric neurons (Raper, 1979; Graubard et al., 1983; Hartline et al., 1988; Manor et al., 1997). Yet, many pyloric synapses have a strong spike-mediated component. Interestingly, when we played back the LP neuron realistic waveform at the amplitude which it was recorded, we saw little graded transmission in control conditions yet this synaptic component was clearly present in proctolin (Fig. 7C). It is therefore possible that under different modulatory conditions one or the other component of synaptic transmission is dominant in affecting the total synaptic strength.

It is common for neuromodulators to change the activity waveform of bursting neurons (Marder and Thirumalai, 2002). Proctolin, for example, increases the amplitude of the LP neuron burst waveform and its spike frequency (Hooper and Marder, 1984; Nusbaum and Marder, 1989b). The LP to PD synapse is 
significantly enhanced if the amplitude of the LP waveform is increased, independent of any direct actions of proctolin on the synapse (Fig. 7). This enhancement is due both to the increase in strength of the graded component with presynaptic voltage amplitude and because the spike-mediated component is enhanced when the baseline membrane potential is increased. However, proctolin also directly enhances these two synaptic components. Thus, during ongoing oscillations, the proctolin enhancement of the LP to PD synapse results from a combination of direct modulation as well as enhanced release due to the change in the presynaptic waveform amplitude and shape.

\section{Network consequences of proctolin enhancement of the LP to PD synapse}

Many oscillatory networks involve pacemaker neurons that receive rhythmic inhibitory feedback (Ramirez et al., 2004; Marder and Bucher, 2007). Previous experimental studies of the pyloric network have shown that the inhibitory LP to PD feedback synapse may have little effect on the average pyloric cycle period (Mamiya and Nadim, 2004; Nadim et al., 2011) even if the synapse is drastically strengthened by a neuromodulator (Thirumalai et al., 2006). These studies proposed that such feedback inhibition may act to stabilize the pyloric cycle period in response to perturbing inputs. Here we show that the variability of the pyloric cycle period is significantly decreased by proctolin and that this stabilizing effect is a direct consequence of the pyloric modulation of the LP to PD synapse (Fig. 9). Feedback inhibition plays an important role in generating and regulating oscillatory networks (Hentall and White, 1997; Mann et al., 2005; Martinez, 2005; Blitz and Nusbaum, 2008) and has been shown to be involved in promoting stability and synchronization in hippocampal gamma oscillations (Stenkamp et al., 2001; Bartos et al., 2007). Our findings therefore indicate that neuromodulation of feedback inhibition may be a regulatory mechanism for producing stable oscillations in a variety of networks ranging from the respiratory network to thalamocortical oscillations during sleep.

\section{References}

Abbott LF, Varela JA, Sen K, Nelson SB (1997) Synaptic depression and cortical gain control. Science 275:220-224.

Alle H, Geiger JR (2006) Combined analog and action potential coding in hippocampal mossy fibers. Science 311:1290-1293.

Angstadt JD, Calabrese RL (1991) Calcium currents and graded synaptic transmission between heart interneurons of the leech. J Neurosci 11:746-759.

Ayali A, Johnson BR, Harris-Warrick RM (1998) Dopamine modulates graded and spike-evoked synaptic inhibition independently at single synapses in pyloric network of lobster. J Neurophysiol 79:2063-2069.

Baimoukhametova DV, Hewitt SA, Sank CA, Bains JS (2004) Dopamine modulates use-dependent plasticity of inhibitory synapses. J Neurosci 24:5162-5171.

Baines RA, Lange AB, Downer RG (1990) Proctolin in the innervation of the locust mandibular closer muscle modulates contractions through the elevation of inositol trisphosphate. J Comp Neurol 297:479-486.

Barrière G, Tartas M, Cazalets JR, Bertrand SS (2008) Interplay between neuromodulator-induced switching of short-term plasticity at sensorimotor synapses in the neonatal rat spinal cord. J Physiol 586:1903-1920.

Bartos M, Vida I, Jonas P (2007) Synaptic mechanisms of synchronized gamma oscillations in inhibitory interneuron networks. Nat Rev Neurosci 8:45-56.

Bevan S, Parker D (2004) Metaplastic facilitation and ultrastructural changes in synaptic properties are associated with long-term modulation of the lamprey locomotor network. J Neurosci 24:9458-9468.

Bishop CA, Krouse ME, Wine JJ (1991) Peptide cotransmitter potentiates calcium channel activity in crayfish skeletal muscle. J Neurosci 11:269-276.

Blitz DM, Nusbaum MP (2008) State-dependent presynaptic inhibition regulates central pattern generator feedback to descending inputs. J Neurosci 28:9564-9574.

Blitz DM, Beenhakker MP, Nusbaum MP (2004) Different sensory systems share projection neurons but elicit distinct motor patterns. J Neurosci 24:11381-11390.

Blitz DM, White RS, Saideman SR, Cook A, Christie AE, Nadim F, Nusbaum MP (2008) A newly identified extrinsic input triggers a distinct gastric mill rhythm via activation of modulatory projection neurons. J Exp Biol 211:1000-1011.

Bristol AS, Fischer TM, Carew TJ (2001) Combined effects of intrinsic facilitation and modulatory inhibition of identified interneurons in the siphon withdrawal circuitry of Aplysia. J Neurosci 21:8990-9000.

Cartling B (2004) Neuromodulatory control of neocortical microcircuits with activity-dependent short-term synaptic depression. J Biol Phys 30:261-284

Deeg KE (2009) Synapse-specific homeostatic mechanisms in the hippocampus. J Neurophysiol 101:503-506.

Eisen JS, Marder E (1982) Mechanisms underlying pattern generation in lobster stomatogastric ganglion as determined by selective inactivation of identified neurons. III. Synaptic connections of electrically coupled pyloric neurons. J Neurophysiol 48:1392-1415.

Eisen JS, Marder E (1984) A mechanism for production of phase shifts in a pattern generator. J Neurophysiol 51:1375-1393.

Fischer TM, Zucker RS, Carew TJ (1997a) Activity-dependent potentiation of synaptic transmission from L30 inhibitory interneurons of Aplysia depends on residual presynaptic $\mathrm{Ca}^{2+}$ but not on postsynaptic $\mathrm{Ca}^{2+}$. J Neurophysiol 78:2061-2071.

Fischer TM, Blazis DE, Priver NA, Carew TJ (1997b) Metaplasticity at identified inhibitory synapses in Aplysia. Nature 389:860-865.

Flamm RE, Fickbohm D, Harris-Warrick RM (1987) cAMP elevation modulates physiological activity of pyloric neurons in the lobster stomatogastric ganglion. J Neurophysiol 58:1370-1386.

Gil Z, Connors BW, Amitai Y (1997) Differential regulation of neocortical synapses by neuromodulators and activity. Neuron 19:679-686.

Giocomo LM, Hasselmo ME (2007) Neuromodulation by glutamate and acetylcholine can change circuit dynamics by regulating the relative influence of afferent input and excitatory feedback. Mol Neurobiol 36:184-200.

Golowasch J, Marder E (1992) Proctolin activates an inward current whose voltage dependence is modified by extracellular $\mathrm{Ca}^{2+}$. J Neurosci 12:810-817.

Golowasch J, Buchholtz F, Epstein IR, Marder E (1992) Contribution of individual ionic currents to activity of a model stomatogastric ganglion neuron. J Neurophysiol 67:341-349.

Graubard K, Raper JA, Hartline DK (1983) Graded synaptic transmission between identified spiking neurons. J Neurophysiol 50:508-521.

Harris-Warrick RM, Johnson BR, Peck JH, Kloppenburg P, Ayali A, Skarbinski J (1998) Distributed effects of dopamine modulation in the crustacean pyloric network. Ann N Y Acad Sci 860:155-167.

Hartline DK, Russell DF, Raper JA, Graubard K (1988) Special cellular and synaptic mechanisms in motor pattern generation. Comp Biochem Physiol C 91:115-131.

Hempel CM, Vincent P, Adams SR, Tsien RY, Selverston AI (1996) Spatiotemporal dynamics of cyclic AMP signals in an intact neural circuit. Nature 384:166-169.

Hentall ID, White TR (1997) Evidence for rhythmic firing being caused by feedback inhibition in pinch-inhibited raphe magnus neurons. Brain Res 745:348-351.

Hooper SL (1997) Phase maintenance in the pyloric pattern of the lobster (Panulirus interruptus) stomatogastric ganglion. J Comput Neurosci 4:191-205.

Hooper SL, Marder E (1984) Modulation of a central pattern generator by two neuropeptides, proctolin and FMRFamide. Brain Res 305:186-191.

Hooper SL, Marder E (1987) Modulation of the lobster pyloric rhythm by the peptide proctolin. J Neurosci 7:2097-2112.

Hurley LM, Graubard K (1998) Pharmacologically and functionally distinct calcium currents of stomatogastric neurons. J Neurophysiol 79:2070-2081.

Ivanov AI, Calabrese RL (2003) Modulation of spike-mediated synaptic transmission by presynaptic background $\mathrm{Ca}^{2+}$ in leech heart interneurons. J Neurosci 23:1206-1218.

Ivanov AI, Calabrese RL (2006a) Graded inhibitory synaptic transmission 
between leech interneurons: assessing the roles of two kinetically distinct low-threshold Ca currents. J Neurophysiol 96:218-234.

Ivanov AI, Calabrese RL (2006b) Spike-mediated and graded inhibitory synaptic transmission between leech interneurons: evidence for shared release sites. J Neurophysiol 96:235-251.

Johnson BR, Kloppenburg P, Harris-Warrick RM (2003) Dopamine modulation of calcium currents in pyloric neurons of the lobster stomatogastric ganglion. J Neurophysiol 90:631-643.

Johnson BR, Schneider LR, Nadim F, Harris-Warrick RM (2005) Dopamine modulation of phasing of activity in a rhythmic motor network: contribution of synaptic and intrinsic modulatory actions. J Neurophysiol 94:3101-3111.

Johnson BR, Brown JM, Kvarta MD, Lu JY, Schneider LR, Nadim F, HarrisWarrick RM (2011) Differential modulation of synaptic strength and timing regulate synaptic efficacy in a motor network. J Neurophysiol 105:293-304.

Johnson EC, Bohn LM, Barak LS, Birse RT, Nässel DR, Caron MG, Taghert PH (2003) Identification of Drosophila neuropeptide receptors by G protein-coupled receptors-beta-arrestin2 interactions. J Biol Chem 278:52172-52178.

Lee JH, Gomora JC, Cribbs LL, Perez-Reyes E (1999) Nickel block of three cloned T-type calcium channels: low concentrations selectively block alpha1H. Biophys J 77:3034-3042.

Logsdon S, Johnstone AF, Viele K, Cooper RL (2006) Regulation of synaptic vesicles pools within motor nerve terminals during short-term facilitation and neuromodulation. J Appl Physiol 100:662-671.

Lu J, Dalton JF 4th, Stokes DR, Calabrese RL (1997) Functional role of $\mathrm{Ca}^{2+}$ currents in graded and spike-mediated synaptic transmission between leech heart interneurons. J Neurophysiol 77:1779-1794.

Mamiya A, Nadim F (2004) Dynamic interaction of oscillatory neurons coupled with reciprocally inhibitory synapses acts to stabilize the rhythm period. J Neurosci 24:5140-5150.

Mamiya A, Nadim F (2005) Target-specific short-term dynamics are important for the function of synapses in an oscillatory neural network. J Neurophysiol 94:2590-2602.

Mamiya A, Manor Y, Nadim F (2003) Short-term dynamics of a mixed chemical and electrical synapse in a rhythmic network. J Neurosci 23:9557-9564.

Mann EO, Suckling JM, Hajos N, Greenfield SA, Paulsen O (2005) Perisomatic feedback inhibition underlies cholinergically induced fast network oscillations in the rat hippocampus in vitro. Neuron 45:105-117.

Manor Y, Nadim F (2001) Synaptic depression mediates bistability in neuronal networks with recurrent inhibitory connectivity. J Neurosci 21:9460-9470.

Manor Y, Nadim F, Abbott LF, Marder E (1997) Temporal dynamics of graded synaptic transmission in the lobster stomatogastric ganglion. J Neurosci 17:5610-5621.

Marder E, Bucher D (2007) Understanding circuit dynamics using the stomatogastric nervous system of lobsters and crabs. Annu Rev Physiol 69:291-316.

Marder E, Thirumalai V (2002) Cellular, synaptic and network effects of neuromodulation. Neural Netw 15:479-493.

Marder E, Hooper SL, Siwicki KK (1986) Modulatory action and distribution of the neuropeptide proctolin in the crustacean stomatogastric nervous system. J Comp Neurol 243:454-467.

Martinez D (2005) Oscillatory synchronization requires precise and balanced feedback inhibition in a model of the insect antennal lobe. Neural Comput 17:2548-2570.

Miller JP, Selverston AI (1982) Mechanisms underlying pattern generation in lobster stomatogastric ganglion as determined by selective inactivation of identified neurons. II. Oscillatory properties of pyloric neurons. J Neurophysiol 48:1378-1391.

Nadim F, Zhao S, Zhou L, Bose A (2011) Inhibitory feedback promotes stability in an oscillatory network. J Neural Eng, in press.

Nicholls J, Wallace BG (1978) Quantal analysis of transmitter release at an inhibitory synapse in the central nervous system of the leech. J Physiol 281:171-185.

Norris BJ, Coleman MJ, Nusbaum MP (1996) Pyloric motor pattern modification by a newly identified projection neuron in the crab stomatogastric nervous system. J Neurophysiol 75:97-108.

Nusbaum MP (2002) Regulating peptidergic modulation of rhythmically active neural circuits. Brain Behav Evol 60:378-387.
Nusbaum MP, Beenhakker MP (2002) A small-systems approach to motor pattern generation. Nature 417:343-350.

Nusbaum MP, Marder E (1989a) A modulatory proctolin-containing neuron (MPN). I. Identification and characterization. J Neurosci 9:15911599.

Nusbaum MP, Marder E (1989b) A modulatory proctolin-containing neuron (MPN). II. State-dependent modulation of rhythmic motor activity. J Neurosci 9:1600-1607.

Nusbaum MP, Blitz DM, Swensen AM, Wood D, Marder E (2001) The roles of co-transmission in neural network modulation. Trends Neurosci 24:146-154.

Otsu Y, Shahrezaei V, Li B, Raymond LA, Delaney KR, Murphy TH (2004) Competition between phasic and asynchronous release for recovered synaptic vesicles at developing hippocampal autaptic synapses. J Neurosci 24:420-433.

Pan ZH, Hu HJ, Perring P, Andrade R (2001) T-type Ca(2+) channels mediate neurotransmitter release in retinal bipolar cells. Neuron 32:89-98.

Parker D (2003) Activity-dependent feedforward inhibition modulates synaptic transmission in a spinal locomotor network. J Neurosci 23:1108511093.

Parker D, Gilbey T (2007) Developmental differences in neuromodulation and synaptic properties in the lamprey spinal cord. Neuroscience 145:142-152.

Perez-Reyes E (2003) Molecular physiology of low-voltage-activated $t$-type calcium channels. Physiol Rev 83:117-161.

Rabbah P, Nadim F (2005) Synaptic dynamics do not determine proper phase of activity in a central pattern generator. J Neurosci 25:11269-11278.

Rabbah P, Nadim F (2007) Distinct synaptic dynamics of heterogeneous pacemaker neurons in an oscillatory network. J Neurophysiol 97:2239-2253.

Rabbah P, Golowasch J, Nadim F (2005) Effect of electrical coupling on ionic current and synaptic potential measurements. J Neurophysiol 94:519-530.

Ramirez JM, Tryba AK, Peña F (2004) Pacemaker neurons and neuronal networks: an integrative view. Curr Opin Neurobiol 14:665-674.

Raper JA (1979) Nonimpulse-mediated synaptic transmission during the generation of a cyclic motor program. Science 205:304-306.

Sakurai A, Katz PS (2003) Spike timing-dependent serotonergic neuromodulation of synaptic strength intrinsic to a central pattern generator circuit. J Neurosci 23:10745-10755.

Sakurai A, Katz PS (2009) State-, timing-, and pattern-dependent neuromodulation of synaptic strength by a serotonergic interneuron. J Neurosci 29:268-279.

Sakurai A, Darghouth NR, Butera RJ, Katz PS (2006) Serotonergic enhancement of a 4-AP-sensitive current mediates the synaptic depression phase of spike timing-dependent neuromodulation. J Neurosci 26:2010-2021.

Shu Y, Hasenstaub A, Duque A, Yu Y, McCormick DA (2006) Modulation of intracortical synaptic potentials by presynaptic somatic membrane potential. Nature 441:761-765.

Soto-Treviño C, Rabbah P, Marder E, Nadim F (2005) Computational model of electrically coupled, intrinsically distinct pacemaker neurons. J Neurophysiol 94:590-604.

Stenkamp K, Palva JM, Uusisaari M, Schuchmann S, Schmitz D, Heinemann U, Kaila K (2001) Enhanced temporal stability of cholinergic hippocampal gamma oscillations following respiratory alkalosis in vitro. J Neurophysiol 85:2063-2069.

Stout AK, Li-Smerin Y, Johnson JW, Reynolds IJ (1996) Mechanisms of glutamate-stimulated $\mathrm{Mg}^{2+}$ influx and subsequent $\mathrm{Mg}^{2+}$ efflux in rat forebrain neurones in culture. J Physiol 492:641-657.

Swensen AM, Marder E (2000) Multiple peptides converge to activate the same voltage-dependent current in a central pattern-generating circuit. J Neurosci 20:6752-6759.

Swensen AM, Marder E (2001) Modulators with convergent cellular actions elicit distinct circuit outputs. J Neurosci 21:4050-4058.

Thirumalai V, Prinz AA, Johnson CD, Marder E (2006) Red pigment concentrating hormone strongly enhances the strength of the feedback to the pyloric rhythm oscillator but has little effect on pyloric rhythm period. J Neurophysiol 95:1762-1770.

Tohidi V, Nadim F (2009) Membrane resonance in bursting pacemaker neurons of an oscillatory network is correlated with network frequency. J Neurosci 29:6427-6435. 
Tseng HA, Nadim F (2010) The membrane potential waveform of bursting pacemaker neurons is a predictor of their preferred frequency and the network cycle frequency. J Neurosci 30:10809-10819.

Tsodyks M, Uziel A, Markram H (2000) Synchrony generation in recurrent networks with frequency-dependent synapses. J Neurosci 20:RC50(1-5).

Warzecha AK, Kurtz R, Egelhaaf M (2003) Synaptic transfer of dynamic motion information between identified neurons in the visual system of the blowfly. Neuroscience 119:1103-1112.

Weaver AL, Hooper SL (2003) Relating network synaptic connectivity and network activity in the lobster (Panulirus interruptus) pyloric network. J Neurophysiol 90:2378-2386.

Wegener C, Nässel DR (2000) Peptide-induced $\mathrm{Ca}(2+)$ movements in a tonic insect muscle: effects of proctolin and periviscerokinin-2. J Neurophysiol 84:3056-3066.

Weimann JM, Meyrand P, Marder E (1991) Neurons that form multiple pattern generators: identification and multiple activity patterns of gastric/ pyloric neurons in the crab stomatogastric system. J Neurophysiol 65:111-122.

Zirpel L, Baldwin D, Graubard K (1993) Nickel induces oscillatory behavior and enhanced synaptic and electrotonic transmission between stomatogastric neurons of Panulirus interruptus. Brain Res 617:205-213.

Zucker RS, Regehr WG (2002) Short-term synaptic plasticity. Annu Rev Physiol 64:355-405. 